University of New Mexico

UNM Digital Repository

Mathematics and Statistics Faculty and Staff

Publications

Academic Department Resources

2-2018

NS-Cross Entropy-Based MAGDM under Single-Valued

Neutrosophic Set Environment

Florentin Smarandache

Surapati Pramanik

Shyamal Dalapati

Shariful Alam

Tapan Kumar Roy

Follow this and additional works at: https://digitalrepository.unm.edu/math_fsp

Part of the Mathematics Commons, Other Applied Mathematics Commons, Partial Differential Equations Commons, and the Special Functions Commons 


\title{
NS-Cross Entropy-Based MAGDM under Single-Valued Neutrosophic Set Environment
}

\author{
Surapati Pramanik 1,*, Shyamal Dalapati ${ }^{2}$, Shariful Alam ${ }^{2}$, Florentin Smarandache ${ }^{3}$ and \\ Tapan Kumar Roy ${ }^{2}$ \\ 1 Department of Mathematics, Nandalal Ghosh B.T. College, Panpur, P.O.-Narayanpur, District-North 24 \\ Parganas, Bhatpara 743126, West Bengal, India \\ 2 Department of Mathematics, Indian Institute of Engineering Science and Technology, Shibpur, P.O.-Botanic \\ Garden, Howrah 711103, West Bengal, India; shyamal.rs2015@math.iiests.ac.in (S.D.); \\ salam@math.iiests.ac.in (S.A.); tkroy@math.iiests.ac.in (T.K.R.) \\ 3 Department of Mathematics \& Science, University of New Mexico, 705 Gurley Ave., Gallup, NM 87301, \\ USA; smarand@unm.edu \\ * Correspondence: surapati.math@gmail.com; Tel.: +91-9477035544
}

Received: 29 December 2017; Accepted: 6 February 2018; Published: 9 February 2018

\begin{abstract}
A single-valued neutrosophic set has king power to express uncertainty characterized by indeterminacy, inconsistency and incompleteness. Most of the existing single-valued neutrosophic cross entropy bears an asymmetrical behavior and produces an undefined phenomenon in some situations. In order to deal with these disadvantages, we propose a new cross entropy measure under a single-valued neutrosophic set (SVNS) environment, namely NS-cross entropy, and prove its basic properties. Also we define weighted NS-cross entropy measure and investigate its basic properties. We develop a novel multi-attribute group decision-making (MAGDM) strategy that is free from the drawback of asymmetrical behavior and undefined phenomena. It is capable of dealing with an unknown weight of attributes and an unknown weight of decision-makers. Finally, a numerical example of multi-attribute group decision-making problem of investment potential is solved to show the feasibility, validity and efficiency of the proposed decision-making strategy.
\end{abstract}

Keywords: neutrosophic set; single-valued neutrosophic set; NS-cross entropy measure; multi-attribute group decision-making

\section{Introduction}

To tackle the uncertainty and modeling of real and scientific problems, Zadeh [1] first introduced the fuzzy set by defining membership measure in 1965. Bellman and Zadeh [2] contributed important research on fuzzy decision-making using max and min operators. Atanassov [3] established the intuitionistic fuzzy set (IFS) in 1986 by adding non-membership measure as an independent component to the fuzzy set. Theoretical and practical applications of IFSs in multi-criteria decision-making (MCDM) have been reported in the literature [4-12]. Zadeh [13] introduced entropy measure in the fuzzy environment. Burillo and Bustince [14] proposed distance measure between IFSs and offered an axiomatic definition of entropy measure. In the IFS environment, Szmidt and Kacprzyk [15] proposed a new entropy measure based on geometric interpretation of IFS. Wei et al. [16] developed an entropy measure for interval-valued intuitionistic fuzzy set (IVIFS) and presented its applications in pattern recognition and MCDM. Li [17] presented a new multi-attribute decision-making (MADM) strategy combining entropy and Technique for Order Preference by Similarity to Ideal Solution (TOPSIS) in an IVIFS environment. Shang and Jiang [18] introduced the cross entropy in the fuzzy environment. Vlachos and Sergiadis [19] presented intuitionistic fuzzy cross entropy by extending fuzzy cross entropy [18]. Ye [20] defined a new cross 
entropy under an IVIFS environment and presented an optimal decision-making strategy. Xia and $\mathrm{Xu}$ [21] put forward a new entropy and a cross entropy and employed them for multi-attribute criteria group decision-making (MAGDM) strategy under an IFS environment. Tong and Yu [22] defined cross entropy under an IVIFS environment and applied it to MADM problems.

The study of uncertainty took a new direction after the publication of the neutrosophic set (NS) [23] and single-valued neutrosophic set (SVNS) [24]. SVNS appeals more to researchers for its applicability in decision-making [25-54], conflict resolution [55], educational problems [56,57], image processing [58-60], cluster analysis [61,62], social problems [63,64], etc. The research on SVNS gained momentum after the inception of the international journal "Neutrosophic Sets and Systems". Combining with the neutrosophic set, a number of hybrid neutrosophic sets such as the neutrosophic soft set [65-72], the neutrosophic soft expert set [73-75], the neutrosophic complex set [76], the rough neutrosophic set [77-86], the rough neutrosophic tri complex set [87], the neutrosophic rough hyper complex set [88], the neutrosophic hesitant fuzzy sets/multi-valued neutrosophic set [89-97], the bipolar neutrosophic set [98-103], the rough bipolar neutrosophic set [104], the neutrosophic cubic set [105-113], and the neutrosophic cubic soft set [114,115] has been reported in the literature. Wang et al. [116] defined the interval neutrosophic set (INS). Different interval neutrosophic hybrid sets and their theoretical development and applications have been reported in the literature, such as the interval-valued neutrosophic soft set [117], the interval neutrosophic complex set [118], the interval neutrosophic rough set [119-121], and the interval neutrosophic hesitant fuzzy set [122]. Other extensions of neutrosophic sets, such as trapezoidal neutrosophic sets $[123,124]$, normal neutrosophic sets [125], single-valued neutrosophic linguistic sets [126], interval neutrosophic linguistic sets [127,128], simplified neutrosophic linguistic sets [129], single-valued neutrosophic trapezoid linguistic sets [130], interval neutrosophic uncertain linguistic sets [131-133], neutrosophic refined sets [134-139], linguistic refined neutrosophic sets [140] bipolar neutrosophic refined sets [141], and dynamic single-valued neutrosophic multi-sets [142] have been proposed to enrich the study of neutrosophics. So the field of neutrosophic study has been steadily developing.

Majumdar and Samanta [143] defined an entropy measure and presented an MCDM strategy under SVNS environment. Ye [144] proposed cross entropy measure under the single-valued neutrosophic set environment, which is not symmetric straight forward and bears undefined phenomena. To overcome the asymmetrical behavior of the cross entropy measure, Ye [144] used a symmetric discrimination information measure for single-valued neutrosophic sets. Ye [145] defined cross entropy measures for SVNSs to overcome the drawback of undefined phenomena of the cross entropy measure [144] and proposed a MCDM strategy.

The aforementioned applications of cross entropy [144,145] can be effective in dealing with neutrosophic MADM problems. However, they also bear some limitations, which are outlined below:

i. The strategies $[144,145]$ are capable of solving neutrosophic MADM problems that require the criterion weights to be completely known. However, it can be difficult and subjective to offer exact criterion weight information due to neutrosophic nature of decision-making situations.

ii. The strategies $[144,145]$ have a single decision-making structure, and not enough attention is paid to improving robustness when processing the assessment information.

iii. The strategies $[144,145]$ cannot deal with the unknown weight of the decision-makers.

\section{Research gap:}

MAGDM strategy based on cross entropy measure with unknown weight of attributes and unknown weight of decision-makers.

This study answers the following research questions:

i. Is it possible to define a new cross entropy measure that is free from asymmetrical phenomena and undefined behavior?

ii. Is it possible to define a new weighted cross entropy measure that is free from the asymmetrical phenomena and undefined behavior? 
iii. Is it possible to develop a new MAGDM strategy based on the proposed cross entropy measure in single-valued neutrosophic set environment, which is free from the asymmetrical phenomena and undefined behavior?

iv. Is it possible to develop a new MAGDM strategy based on the proposed weighted cross entropy measure in the single-valued neutrosophic set environment that is free from the asymmetrical phenomena and undefined behavior?

v. How do we assign unknown weight of attributes?

vi. How do we assign unknown weight of decision-makers?

\section{Motivation:}

The above-mentioned analysis describes the motivation behind proposing a comprehensive NS-cross entropy-based strategy for tackling MAGDM under the neutrosophic environment. This study develops a novel NS-cross entropy-based MAGDM strategy that can deal with multiple decision-makers and unknown weight of attributes and unknown weight of decision-makers and free from the drawbacks that exist in [144,145].

The objectives of the paper are:

1. To define a new cross entropy measure and prove its basic properties, which are free from asymmetrical phenomena and undefined behavior.

2. To define a new weighted cross measure and prove its basic properties, which are free from asymmetrical phenomena and undefined behavior.

3. To develop a new MAGDM strategy based on weighted cross entropy measure under single-valued neutrosophic set environment.

4. To develop a technique to incorporate unknown weight of attributes and unknown weight of decision-makers in the proposed NS-cross entropy-based MAGDM under single-valued neutrosophic environment.

To fill the research gap, we propose NS-cross entropy-based MAGDM, which is capable of dealing with multiple decision-makers with unknown weight of the decision-makers and unknown weight of the attributes.

The main contributions of this paper are summarized below:

1. We define a new NS-cross entropy measure and prove its basic properties. It is straightforward symmetric and it has no undefined behavior.

2. We define a new weighted NS-cross entropy measure in the single-valued neutrosophic set environment and prove its basic properties. It is straightforward symmetric and it has no undefined behavior.

3. In this paper, we develop a new MAGDM strategy based on weighted NS cross entropy to solve MAGDM problems with unknown weight of the attributes and unknown weight of decision-makers.

4. Techniques to determine unknown weight of attributes and unknown weight of decisions makers are proposed in the study.

The rest of the paper is presented as follows: Section 2 describes some concepts of SVNS. In Section 3 we propose a new cross entropy measure between two SVNS and investigate its properties. In Section 4, we develop a novel MAGDM strategy based on the proposed NS-cross entropy with SVNS information. In Section 5 an illustrative example is solved to demonstrate the applicability and efficiency of the developed MAGDM strategy under SVNS environment. In Section 6 we present comparative study and discussion. Section 7 offers conclusions and the future scope of research.

\section{Preliminaries}

This section presents a short list of mostly known definitions pertaining to this paper.

Definition 1 [23] NS. Let $U$ be a space of points (objects) with a generic element in $U$ denoted by $u$, i.e., $u \in$ $U$. A neutrosophic set $A$ in $U$ is characterized by truth-membership measure $T_{A}(u)$, indeterminacy- 
membership measure $I_{A}(u)$ and falsity-membership measure $F_{A}(u)$, where $T_{A}(u), I_{A}(u), F_{A}(u)$ are the measures from $U$ to $]^{-} 0,1^{+}\left[\text {i.e., } T_{A}(u), I_{A}(u), F_{A}(u): U \rightarrow\right]^{-} 0,1^{+}\left[N S\right.$ can be expressed as $A=\left\{\left\langle u ;\left(T_{A}(u)\right.\right.\right.$ , $\left.\left.I_{A}(u), F_{A}(u)\right): \forall u \in U\right\}$. Since $T_{A}(u), I_{A}(u), F_{A}(u)$ are the subsets of $]^{-0}, 1^{+}\left[\right.$there the sum $\left(T_{A}(u)+\right.$ $I_{A}(u)+F_{A}(u)$ ) lies between -0 and $3^{+}$.

Example 1. Suppose that $U=\left\{u_{1}, u_{2}, u_{3}, \ldots\right\}$ be the universal set. Let $R_{1}$ be any neutrosophic set in $U$. Then $R_{1}$ expressed as $R_{1}=\left\{<u_{1} ;(0.6,0.3,0.4)>: u_{1} \in U\right\}$.

Definition 2 [24] SVNS. Assume that $U$ be a space of points (objects) with generic elements $u \in U$. A SVNS $H$ in $U$ is characterized by a truth-membership measure $T_{H}(u)$, an indeterminacy-membership measure $I_{H}(u)$, and a falsity-membership measure $F_{H}(u)$, where $T_{H}(u), I_{H}(u), F_{H}(u) \in[0,1]$ for each point $u$ in $U$. Therefore, $a$ SVNS A can be expressed as $H=\left\{u,\left(T_{H}(u), I_{H}(u), F_{H}(u)\right) \mid \forall u \in U\right\}$, whereas, the sum of $T_{H}(u), I_{H}(u)$ and $F_{H}(u)$ satisfy the condition $0 \leq T_{H}(u)+I_{H}(u)+F_{H}(u) \leq 3$ and $H(u)=\left\langle\left(T_{H}(u), I_{H}(u), F_{H}(u)>\right.\right.$ call a single-valued neutrosophic number (SVNN).

Example 2. Suppose that $U=\left\{u_{1}, u_{2}, u_{3}, \ldots\right\}$ be the universal set. A SVNS H in $U$ can be expressed as: $H=\left\{u_{1}\right.$, $\left.(0.7,0.3,0.5) \mid u_{1} \in U\right\}$ and SVNN presented $H=<0.7,0.3,0.5>$.

Definition 3 [24] Inclusion of SVNSs. The inclusion of any two SVNS sets $\mathrm{H}_{1}$ and $\mathrm{H}_{2}$ in $U$ is denoted by $\mathrm{H}_{1} \subseteq \mathrm{H}_{2}$ and defined as follows:

$$
H_{1} \subseteq H_{2}, T_{H_{1}}(u) \leq T_{H_{2}}(u), I_{H_{1}}(u) \geq I_{H_{2}}(u), F_{H_{1}}(u) \geq F_{H_{2}}(u) \text { iff for all } u \in U .
$$

Example 3. Let $H_{1}$ and $H_{2}$ be any two SVNNs in U presented as follows: $H_{1}=<(0.7,0.3,0.5)>$ and $H_{2}=<(0.8$, $0.2,0.4)>$ for all $u \in U$. Using the property of inclusion of two SVNNs, we conclude that $H_{1} \subseteq H_{2}$.

Definition 4 [24] Equality of two SVNSs. The equality of any two SVNS $H_{1}$ and $H_{2}$ in $U$ denoted by $H_{1}=$ $\mathrm{H}_{2}$ and defined as follows:

$$
T_{H_{1}}(u)=T_{H_{2}}(u), I_{H_{1}}(u)=I_{H_{2}}(u) \text { and } F_{H_{1}}(u)=F_{H_{2}}(u) \text { for all } u \in U \text {. }
$$

Definition 5 Complement of any SVNSs. The complement of any SVNS $H$ in $U$ denoted by $H^{c}$ and defined as follows:

$$
H^{c}=\left\{u, 1-T_{H}, 1-I_{H}, 1-F_{H} \mid u \in U\right\} .
$$

Example 4. Let $H$ be any SVNN in $U$ presented as follows: $H=\langle(0.7,0.3,0.5)\rangle$. Then compliment of $H$ is obtained as $H^{c}=\langle(0.3,0.7,0.5)\rangle$.

Definition 6 [24] Union. The union of two single-valued neutrosophic sets $\mathrm{H}_{1}$ and $\mathrm{H}_{2}$ is a neutrosophic set $\mathrm{H}_{3}$ (say) written as

$H_{3}=H_{1} \cup H_{2}$.

$T_{H_{3}}(u)=\max \left\{T_{H_{1}}(u), T_{H_{2}}(u)\right\}, I_{H_{3}}(u)=\min \left\{I_{H_{1}}(u), I_{H_{2}}(u)\right\}, F_{H_{3}}(u)=\min \left\{F_{H_{1}}(u), F_{H_{2}}(u)\right\}, \forall u \in U$.

Example 5. Let $H_{1}$ and $H_{2}$ be two SVNSs in $U$ presented as follows:

$H_{1}=<(0.6,0.3,0.4)>$ and $H_{2}=<(0.7,0.3,0.6)>$. Then union of them is presented as:

$$
H_{1} \cup H_{2}=<(0.7,0.3,0.4)>\text {. }
$$


Definition 7 [24] Intersection. The intersection of two single-valued neutrosophic sets $\mathrm{H}_{1}$ and $\mathrm{H}_{2}$ denoted by $\mathrm{H}_{4}$ and defined as

$$
\begin{aligned}
& H_{4}=H_{1} \cap H_{2} \\
& T_{H_{4}}(u)=\min \left\{T_{H_{1}}(u), T_{H_{2}}(u)\right\}, I_{H_{4}}(u)=\max \left\{I_{H_{1}}(u), I_{H_{2}}(u)\right\} \\
& F_{H_{4}}(u)=\max \left\{F_{H_{1}}(u), F_{H_{2}}(u)\right\}, \forall u \in U .
\end{aligned}
$$

Example 6. Let $\mathrm{H}_{1}$ and $\mathrm{H}_{2}$ be two SVNSs in U presented as follows:

$$
H_{1}=<(0.6,0.3,0.4)>\text { and } H_{2}=<(0.7,0.3,0.6)>\text {. }
$$

Then intersection of $\mathrm{H}_{1}$ and $\mathrm{H}_{2}$ is presented as follows:

$$
H_{1} \cap H_{2}=<(0.6,0.3,0.6)>
$$

\section{NS-Cross Entropy Measure}

In this section, we define a new single-valued neutrosophic cross-entropy measure for measuring the deviation of single-valued neutrosophic variables from an a priori one.

Definition 8 NS-cross entropy measure. Let $H_{1}$ and $H_{2}$ be any two SVNSs in $U=\left\{u_{1}, u_{2}, u_{3}, \ldots, u_{n}\right\}$. Then, the single-valued cross-entropy of $\mathrm{H}_{1}$ and $\mathrm{H}_{2}$ is denoted by $\mathrm{CE}_{N S}\left(\mathrm{H}_{1}, \mathrm{H}_{2}\right)$ and defined as follows:

$$
\begin{aligned}
& \mathrm{CE}_{\mathrm{NS}}\left(\mathrm{H}_{1}, \mathrm{H}_{2}\right)=\frac{1}{2}\left\{\sum_{i=1}^{n} /\left[\frac{2\left|T_{H_{1}}\left(u_{i}\right)-T_{H_{2}}\left(u_{i}\right)\right|}{\sqrt{1+\left|T_{H_{1}}\left(u_{i}\right)\right|^{2}}+\sqrt{1+\left|T_{H_{2}}\left(u_{i}\right)\right|^{2}}}+\frac{2\left|\left(1-T_{H_{1}}\left(u_{i}\right)\right)-\left(1-T_{H_{2}}\left(u_{i}\right)\right)\right|}{\sqrt{1+\mid\left(1-\left.T_{H_{1}}\left(u_{i}\right)\right|^{2}\right.}+\sqrt{1+\left|\left(1-T_{H_{2}}\left(u_{i}\right)\right)\right|^{2}}}\right]+\right.
\end{aligned}
$$

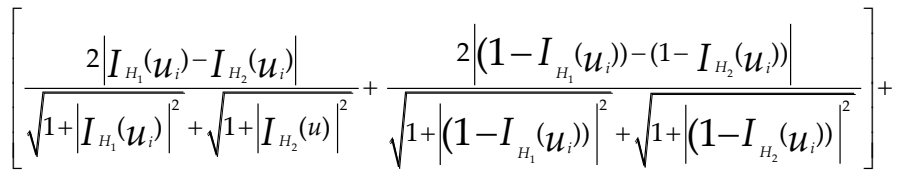

$$
\begin{aligned}
& \left.\left.\left[\frac{2\left|F_{H_{1}}\left(\mathcal{u}_{i}\right)-F_{H_{2}}\left(\mathcal{u}_{i}\right)\right|}{\sqrt{1+\left|F_{H_{1}}\left(\mathcal{u}_{i}\right)\right|^{2}}+\sqrt{1+\left|F_{H_{2}}\left(\mathcal{u}_{i}\right)\right|^{2}}}+\frac{2\left|\left(1-F_{H_{1}}\left(u_{i}\right)\right)-\left(1-F_{H_{2}}\left(u_{i}\right)\right)\right|}{\sqrt{1+\mid\left(1-\left.F_{H_{1}}\left(u_{i}\right)\right|^{2}\right.}+\sqrt{1+\left|\left(1-F_{H_{2}}\left(u_{i}\right)\right)\right|^{2}}}\right]\right)\right\}
\end{aligned}
$$

Example 7. Let $H_{1}$ and $H_{2}$ be two SVNSs in $U$, which are given by $H_{1}=\{u,(0.7,0.3,0.4) \mid u \in U\}$ and $H_{2}=$ $\{u,(0.6,0.4,0.2) \mid u \in U\}$. Using Equation (1), the cross entropy value of $H_{1}$ and $H_{2}$ is obtained as $\mathrm{CE}_{\mathrm{NS}}\left(\mathrm{H}_{1}, \mathrm{H}_{2}\right)=0.707$.

Theorem 1. Single-valued neutrosophic cross entropy $\mathrm{CE}_{\mathrm{NS}}\left(\mathrm{H}_{1}, \mathrm{H}_{2}\right)$ for any two SVNSs $\mathrm{H}_{1}, \mathrm{H}_{2}$, satisfies the following properties:

i. $\mathrm{CE}_{\mathrm{NS}}\left(\mathrm{H}_{1}, \mathrm{H}_{2}\right) \geq 0$.

ii. $\quad \mathrm{CE}_{\mathrm{NS}}\left(\mathrm{H}_{1}, \mathrm{H}_{2}\right)=0$ if and only if $T_{H_{1}}\left(u_{i}\right)=T_{H_{2}}\left(u_{i}\right), I_{H_{1}}\left(u_{i}\right)=I_{H_{2}}\left(u_{i}\right), F_{H_{1}}\left(u_{i}\right)=F_{H_{2}}\left(u_{i}\right), \forall u_{i} \in U$.

iii. $\mathrm{CE}_{\mathrm{NS}}\left(\mathrm{H}_{1}, \mathrm{H}_{2}\right)=\mathrm{CE}_{\mathrm{NS}}\left(\mathrm{H}_{1}^{\mathrm{c}}, \mathrm{H}_{2}^{\mathrm{c}}\right)$

iv. $\mathrm{CE}_{\mathrm{NS}}\left(\mathrm{H}_{1}, \mathrm{H}_{2}\right)=\mathrm{CE}_{\mathrm{NS}}\left(\mathrm{H}_{2}, \mathrm{H}_{1}\right)$

Proof. (i) For all values of $u_{i} \in U,\left|T_{H_{1}}\left(u_{i}\right)\right| \geq 0,\left|T_{H_{2}}\left(\mathcal{u}_{i}\right)\right| \geq 0,\left|T_{H_{1}}\left(u_{i}\right)-T_{H_{2}}\left(u_{i}\right)\right| \geq 0, \sqrt{1+\left|T_{H_{1}}\left(u_{i}\right)\right|^{2}} \geq 0$, $\sqrt{1+\left|T_{H_{2}}\left(\mathcal{u}_{i}\right)\right|^{2}} \geq 0,\left|\left(1-T_{H_{1}}\left(\mathcal{u}_{i}\right)\right)\right| \geq 0,\left|\left(1-T_{H_{2}}\left(\mathcal{u}_{i}\right)\right)\right| \geq 0,\left|\left(1-T_{H_{1}}\left(\mathcal{u}_{i}\right)\right)-\left(1-T_{H_{2}}\left(\mathcal{u}_{i}\right)\right)\right| \geq 0, \sqrt{1+\left|\left(1-T_{H_{1}}\left(\mathcal{u}_{i}\right)\right)\right|^{2}} \geq 0$ ， $\sqrt{1+\mid\left(1-\left.T_{H_{2}}\left(u_{i}\right)\right|^{2}\right.} \geq 0$. 
Then, $\left[\frac{2\left|T_{H_{1}}\left(\mathcal{u}_{i}\right)-T_{H_{2}}\left(\mathcal{u}_{i}\right)\right|}{\sqrt{1+\left|T_{H_{1}}\left(\mathcal{u}_{i}\right)\right|^{2}}+\sqrt{1+\mid T_{H_{2}}\left(\mathcal{U}_{i}\right)^{2}}}+\frac{2\left|\left(1-T_{H_{1}}\left(\mathcal{u}_{i}\right)\right)-\left(1-T_{H_{2}}\left(\mathcal{u}_{i}\right)\right)\right|}{\sqrt{1+\mid\left(1-\left.T_{H_{1}}\left(\mathcal{U}_{i}\right)\right|^{2}\right.}+\sqrt{1+\mid\left(1-\left.T_{H_{2}}\left(\mathcal{U}_{i}\right)\right|^{2}\right.}}\right] \geq 0$.

Similarly,

$$
\left[\frac{2\left|I_{H_{1}}\left(\mathcal{u}_{i}\right)-I_{H_{2}}\left(\mathcal{u}_{i}\right)\right|}{\sqrt{1+\left|I_{H_{1}}\left(\mathcal{U}_{i}\right)\right|^{2}}+\sqrt{1+\left|I_{H_{2}}(u)\right|^{2}}}+\frac{2 \mid\left(1-I_{H_{1}}\left(\mathcal{u}_{i}\right)\right)-\left(1-I_{H_{2}}\left(\mathcal{u}_{i}\right) \mid\right.}{\sqrt{1+\left|\left(1-I_{H_{1}}\left(\mathcal{u}_{i}\right)\right)\right|^{2}}+\sqrt{1+\mid\left(1-\left.I_{H_{2}}\left(\mathcal{U}_{i}\right)\right|^{2}\right.}}\right] \geq 0
$$

and

$\left[\frac{2 \mid F_{H_{1}}\left(\mathcal{u}_{i}\right)-F_{H_{2}}\left(\mathcal{u}_{i} \mid\right.}{\sqrt{1+\left|F_{H_{1}}\left(\mathcal{u}_{i}\right)\right|^{2}}+\sqrt{1+\left|F_{H_{2}}\left(\mathcal{u}_{i}\right)\right|^{2}}}+\frac{2\left(1-F_{H_{1}}\left(\mathcal{u}_{i}\right)\right)-\left(1-F_{H_{2}}\left(\mathcal{u}_{i}\right)\right) \mid}{\sqrt{1+\mid\left(1-\left.F_{H_{1}}\left(\mathcal{u}_{i}\right)\right|^{2}\right.}+\sqrt{1+\mid\left(1-\left.F_{H_{2}}\left(\mathcal{u}_{i}\right)\right|^{2}\right.}}\right] \geq 0$.

Therefore, $\mathrm{CE}_{\mathrm{NS}}\left(\mathrm{H}_{1}, \mathrm{H}_{2}\right) \geq 0$.

Hence complete the proof.

(ii)

$$
\left[\frac{2\left|T_{H_{1}}\left(\mathcal{u}_{i}\right)-T_{H_{2}}\left(\mathcal{u}_{i}\right)\right|}{\sqrt{1+\mid T_{H_{1}}\left(\mathcal{u}_{i}\right)^{2}}+\sqrt{1+\left|T_{H_{2}}\left(\mathcal{U}_{i}\right)\right|^{2}}}+\frac{2 \mid\left(1-T_{H_{1}}\left(\mathcal{U}_{i}\right)\right)-\left(1-T_{H_{2}}\left(\mathcal{u}_{i}\right) \mid\right.}{\sqrt{1+\mid\left(1-T_{H_{1}}\left(\mathcal{u}_{i}\right)\right)^{2}}+\sqrt{1+\left|\left(1-T_{H_{2}}\left(\mathcal{u}_{i}\right)\right)\right|^{2}}}\right]=0, \quad \Leftrightarrow T_{H_{1}}\left(\mathcal{u}_{i}\right)=T_{H_{2}}\left(\mathcal{u}_{i}\right) \quad,
$$

$\left[\frac{2\left|I_{H_{1}}\left(\mathcal{u}_{i}\right)-I_{H_{2}}\left(u_{i}\right)\right|}{\sqrt{1+\left|I_{H_{1}}\left(\mathcal{u}_{i}\right)\right|^{2}}+\sqrt{1+\left|I_{H_{2}}(u)\right|^{2}}}+\frac{2 \mid\left(1-I_{H_{1}}\left(\mathcal{u}_{i}\right)\right)-\left(1-I_{H_{2}}\left(\mathcal{u}_{i}\right) \mid\right.}{\sqrt{1+\mid\left(1-\left.I_{H_{1}}\left(\mathcal{u}_{i}\right)\right|^{2}\right.}+\sqrt{1+\mid\left(1-I_{H_{2}}\left(\mathcal{u}_{i}\right)\right)^{2}}}\right]=0 \quad \Leftrightarrow I_{H_{1}}\left(\mathcal{u}_{i}\right)=I_{H_{2}}\left(\mathcal{u}_{i}\right) \quad, \quad$ and,

$\left[\frac{2\left|F_{H_{1}}\left(\mathcal{u}_{i}\right)-F_{H_{2}}\left(\mathcal{u}_{i}\right)\right|}{\sqrt{1+\left|F_{H_{1}}\left(\mathcal{u}_{i}\right)\right|^{2}}+\sqrt{1+\left|F_{H_{2}}\left(\mathcal{u}_{i}\right)\right|^{2}}}+\frac{2\left|\left(1-F_{H_{1}}\left(\mathcal{U}_{i}\right)\right)-\left(1-F_{H_{2}}\left(\mathcal{u}_{i}\right)\right)\right|}{\sqrt{1+\mid\left(1-F_{H_{1}}\left(\mathcal{u}_{i}\right)\right)^{2}}+\sqrt{1+\mid\left(1-\left.F_{H_{2}}\left(\mathcal{u}_{i}\right)\right|^{2}\right.}}\right]=0, \Leftrightarrow F_{H_{1}}\left(\mathcal{U}_{i}\right)=F_{H_{2}}\left(\mathcal{u}_{i}\right)$

Therefore, $\mathrm{CE}_{\mathrm{NS}}\left(\mathrm{H}_{1}, \mathrm{H}_{2}\right)=0$, iff $T_{H_{1}}\left(\mathcal{u}_{i}\right)=T_{H_{2}}\left(\mathcal{u}_{i}\right), I_{H_{1}}\left(u_{i}\right)=I_{H_{2}}\left(\mathcal{u}_{i}\right), F_{H_{1}}\left(\mathcal{u}_{i}\right)=F_{H_{2}}\left(\mathcal{u}_{i}\right), \quad \forall u_{i} \in U$.

Hence complete the proof.

(iii) Using Definition 5, we obtain the following expression

$$
\begin{aligned}
& \mathrm{CE}_{\mathrm{NS}}\left(\mathrm{H}_{1}^{\mathrm{c}}, \mathrm{H}_{2}^{\mathrm{c}}\right)=\frac{1}{2}\left\{\sum _ { i = 1 } ^ { n } \left(\left[\frac{2\left(1-T_{H_{1}}\left(\mathcal{u}_{i}\right)\right)-\left(1-T_{H_{2}}\left(\mathcal{u}_{i}\right)\right) \mid}{\sqrt{1+\left|\left(1-T_{H_{1}}\left(\mathcal{u}_{i}\right)\right)\right|^{2}}+\sqrt{1+\left(1-\left.T_{H_{2}}\left(\mathcal{U}_{i}\right)\right|^{2}\right.}}+\frac{2\left|T_{H_{1}}\left(\mathcal{u}_{i}\right)-T_{H_{2}}\left(\mathcal{u}_{i}\right)\right|}{\sqrt{1+\mid T_{H_{1}}\left(\mathcal{U}_{i}\right)^{2}}+\sqrt{1+\left|T_{H_{2}}\left(\mathcal{U}_{i}\right)\right|^{2}}}\right]+\right.\right. \\
& {\left[\frac{2 \mid\left(1-I_{H_{1}}\left(\mathcal{U}_{i}\right)\right)-\left(1-I_{H_{2}}\left(\mathcal{U}_{i}\right) \mid\right.}{\sqrt{1+\left(1-I_{H_{1}}\left(\mathcal{u}_{i}\right)\right)^{2}}+\sqrt{1+\mid\left(1-\left.I_{H_{2}}\left(\mathcal{U}_{i}\right)\right|^{2}\right.}}+\frac{2 \mid I_{H_{1}}\left(\mathcal{U}_{i}\right)-I_{H_{2}}\left(\mathcal{u}_{i} \mid\right.}{\sqrt{1+\left|I_{H_{1}}\left(\mathcal{U}_{i}\right)\right|^{2}}+\sqrt{1+\left|I_{H_{2}}(u)\right|^{2}}}\right]+} \\
& \left.\left[\frac{2 \mid\left(1-F_{H_{1}}\left(\mathcal{u}_{i}\right)\right)-\left(1-F_{H_{2}}\left(\mathcal{u}_{i}\right) \mid\right.}{\sqrt{1+\left.\left(1-F_{H_{1}}\left(\mathcal{u}_{i}\right)\right)\right|^{2}}+\sqrt{1+\left.\left(1-F_{H_{2}}\left(\mathcal{u}_{i}\right)\right)\right|^{2}}}+\frac{2\left|F_{H_{1}}\left(\mathcal{u}_{i}\right)-F_{H_{2}}\left(u_{i}\right)\right|}{\sqrt{1+\left|F_{H_{1}}\left(\mathcal{u}_{i}\right)\right|^{2}}+\sqrt{1+\left|F_{H_{2}}\left(\mathcal{u}_{i}\right)\right|^{2}}}\right] /\right\} \\
& =\frac{1}{2}\left\{\sum _ { i = 1 } ^ { n } \left\langle\left[\frac{2\left|T_{H_{1}}\left(\mathcal{u}_{i}\right)-T_{H_{2}}\left(\mathcal{u}_{i}\right)\right|}{\left.\sqrt{1+\mid T_{H_{1}}\left(\mathcal{U}_{i}\right)^{2}}\right|^{2}+\sqrt{1+\mid T_{H_{2}}\left(\mathcal{U}_{i}\right)^{2}}}+\frac{2\left|\left(1-T_{H_{1}}\left(\mathcal{U}_{i}\right)\right)-\left(1-T_{H_{2}}\left(\mathcal{U}_{i}\right)\right)\right|}{\sqrt{1+\mid\left(1-\left.T_{H_{1}}\left(\mathcal{U}_{i}\right)\right|^{2}\right.}+\sqrt{1+\mid\left(1-\left.T_{H_{2}}\left(\mathcal{U}_{i}\right)\right|^{2}\right.}}\right]+\right.\right. \\
& {\left[\frac{2 \mid I_{H_{1}}\left(\mathcal{u}_{i}\right)-I_{H_{2}}\left(\mathcal{u}_{i} \mid\right.}{\sqrt{1+\mid I_{H_{1}}\left(\mathcal{U}_{i}\right)^{2}}+\sqrt{1+\left|I_{H_{2}}(u)\right|^{2}}}+\frac{2\left(1-I_{H_{1}}\left(\mathcal{u}_{i}\right)\right)-\left(1-I_{H_{2}}\left(\mathcal{u}_{i}\right) \mid\right.}{\sqrt{1+\left|\left(1-I_{H_{1}}\left(\mathcal{U}_{i}\right)\right)\right|^{2}}+\sqrt{1+\left(1-\left.I_{H_{2}}\left(\mathcal{u}_{i}\right)\right|^{2}\right.}}\right]+} \\
& \left.\left.\left[\frac{2\left|F_{H_{1}}\left(\mathcal{U}_{i}\right)-F_{H_{2}}\left(\mathcal{U}_{i}\right)\right|}{\sqrt{1+\mid F_{H_{1}}\left(\mathcal{U}_{i}\right)^{2}}+\sqrt{1+\mid F_{H_{2}}\left(\mathcal{U}_{i}\right)^{2}}}+\frac{2\left(1-F_{H_{1}}\left(\mathcal{u}_{i}\right)\right)-\left(1-F_{H_{2}}\left(\mathcal{U}_{i}\right)\right) \mid}{\sqrt{1+\mid\left(1-\left.F_{H_{1}}\left(\mathcal{U}_{i}\right)\right|^{2}\right.}+\sqrt{1+\left(1-\left.F_{H_{2}}\left(\mathcal{U}_{i}\right)\right|^{2}\right.}}\right]\right\}\right\}=\mathrm{CE}_{\mathrm{SN}}\left(\mathrm{H}_{1}, \mathrm{H}_{2}\right)
\end{aligned}
$$


Therefore, $\mathrm{CE}_{\mathrm{NS}}\left(\mathrm{H}_{1}, \mathrm{H}_{2}\right)=\mathrm{CE}_{\mathrm{NS}}\left(\mathrm{H}_{1}^{\mathrm{c}}, \mathrm{H}_{2}^{\mathrm{c}}\right)$.

Hence complete the proof.

(iv) Since, $\quad\left|T_{H_{1}}\left(\mathcal{U}_{i}\right)-T_{H_{2}}\left(\mathcal{U}_{i}\right)\right|=\left|T_{H_{2}}\left(\mathcal{u}_{i}\right)-T_{H_{1}}\left(\mathcal{U}_{i}\right)\right| \quad, \quad\left|I_{H_{1}}\left(\mathcal{U}_{i}\right)-I_{H_{2}}\left(\mathcal{U}_{i}\right)\right|=\left|I_{H_{2}}\left(\mathcal{U}_{i}\right)-I_{H_{1}}\left(\mathcal{U}_{i}\right)\right|$, $\left|F_{H_{1}}\left(\mathcal{U}_{i}\right)-F_{H_{2}}\left(\mathcal{u}_{i}\right)\right|=\left|F_{H_{2}}\left(\mathcal{u}_{i}\right)-F_{H_{1}}\left(\mathcal{u}_{i}\right)\right| \quad, \quad\left|\left(1-T_{H_{1}}\left(\mathcal{u}_{i}\right)\right)-\left(1-T_{H_{2}}\left(\mathcal{u}_{i}\right)\right)\right|=\left|\left(1-T_{H_{2}}\left(\mathcal{u}_{i}\right)\right)-\left(1-T_{H_{1}}\left(\mathcal{u}_{i}\right)\right)\right| \quad$, $\left|\left(1-I_{H_{1}}\left(\mathcal{U}_{i}\right)\right)-\left(1-I_{H_{2}}\left(\mathcal{U}_{i}\right)\right)\right|=\mid\left(1-I_{H_{2}}\left(\mathcal{U}_{i}\right)\right)-\left(1-I_{H_{1}}\left(\mathcal{U}_{i}\right)||,\left(1-F_{H_{1}}\left(\mathcal{U}_{i}\right)\right)-\left(1-F_{H_{2}}\left(\mathcal{U}_{i}\right)|=|\left(1-F_{H_{2}}\left(\mathcal{U}_{i}\right)\right)-\left(1-F_{H_{1}}\left(\mathcal{U}_{i}\right)\right) \mid\right.\right.$, then, $\sqrt{1+\mid T_{H_{1}}\left(\mathcal{U}_{i}\right)^{2}}+\sqrt{1+\mid T_{H_{2}}\left(\mathcal{U}_{i}\right)^{2}}=\sqrt{1+\mid T_{H_{2}}\left(\mathcal{U}_{i}\right)^{2}}+\sqrt{1+\mid T_{H_{1}}\left(\left.\mathcal{U}_{i}\right|^{2}\right.}$ $\sqrt{1+\left|I_{H_{1}}\left(\mathcal{U}_{i}\right)\right|^{2}}+\sqrt{1+\left|I_{H_{2}}\left(\mathcal{U}_{i}\right)\right|^{2}}=\sqrt{1+\left|I_{H_{2}}\left(\mathcal{u}_{i}\right)\right|^{2}}+\sqrt{1+\left|I_{H_{1}}\left(\mathcal{u}_{i}\right)\right|^{2}}$ $\sqrt{1+\left|F_{H_{1}}\left(\mathcal{u}_{i}\right)\right|^{2}}+\sqrt{1+\mid F_{H_{2}}\left(\mathcal{u}_{i}\right)^{2}}=\sqrt{1+\mid F_{H_{2}}\left(\mathcal{U}_{i}\right)^{2}}+\sqrt{1+\mid F_{H_{1}}\left(\left.\mathcal{u}_{i}\right|^{2}\right.}$ $\sqrt{1+\mid\left(1-T_{H_{1}}\left(\mathcal{u}_{i}\right)\right)^{2}}+\sqrt{1+\left|\left(1-T_{H_{2}}\left(\mathcal{u}_{i}\right)\right)\right|^{2}}=\sqrt{1+\mid\left(-\left.T_{H_{2}}\left(\mathcal{U}_{i}\right)\right|^{2}\right.}+\sqrt{1+\mid\left(1-\left.T_{H_{1}}\left(\mathcal{u}_{i}\right)\right|^{2}\right.}$ $\sqrt{1+\left|\left(1-I_{H_{1}}\left(\mathcal{U}_{i}\right)\right)\right|^{2}}+\sqrt{1+\mid\left(1-\left.I_{H_{2}}\left(\mathcal{U}_{i}\right)\right|^{2}\right.}=\sqrt{1+\left|\left(1-I_{H_{2}}\left(\mathcal{U}_{i}\right)\right)\right|^{2}}+\sqrt{1+\mid\left(1-\left.I_{H_{1}}\left(\mathcal{U}_{i}\right)\right|^{2}\right.}$ $\sqrt{1+\mid\left(1-\left.F_{H_{1}}\left(\mathcal{u}_{i}\right)\right|^{2}\right.}+\sqrt{1+\mid\left(1-\left.F_{H_{2}}\left(\mathcal{u}_{i}\right)\right|^{2}\right.}=\sqrt{1+\left|\left(1-F_{H_{2}}\left(\mathcal{u}_{i}\right)\right)\right|^{2}}+\sqrt{1+\left|\left(1-F_{H_{1}}\left(u_{i}\right)\right)\right|^{2}}, \quad \forall u_{i} \in U$.

Therefore, $\mathrm{CE}_{\mathrm{NS}}\left(\mathrm{H}_{1}, \mathrm{H}_{2}\right)=\mathrm{CE}_{\mathrm{NS}}\left(\mathrm{H}_{2}, \mathrm{H}_{1}\right)$.

Hence complete the proof.

Definition 9 Weighted NS-cross entropy measure. We consider the weight $w_{i}(i=1,2, \ldots, n)$ for the element $u_{i}(i=1,2, . ., n)$ with the conditions $\mathrm{w}_{\mathrm{i}} \in[0,1]$ and $\sum_{i=1}^{n} w_{i}=1$.

Then the weighted cross entropy between SVNSs $\mathrm{H}_{1}$ and $\mathrm{H}_{2}$ can be defined as follows:

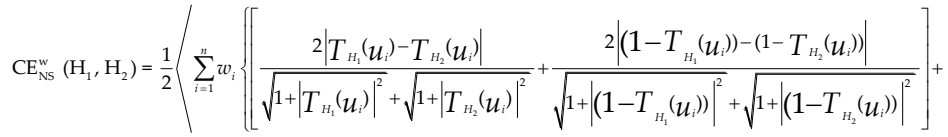

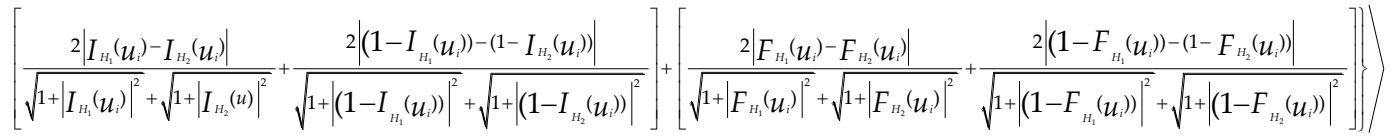

Theorem 2. Single-valued neutrosophic weighted NS-cross-entropy (defined in Equation (2)) satisfies the following properties:
i. $\quad \mathrm{CE}_{\mathrm{NS}}^{\mathrm{w}}\left(\mathrm{H}_{1}, \mathrm{H}_{2}\right) \geq 0$.
ii. $\quad \mathrm{CE}_{\mathrm{NS}}^{\mathrm{w}}\left(\mathrm{H}_{1}, \mathrm{H}_{2}\right)=0$, if and only if $T_{H_{1}}\left(\mathcal{u}_{i}\right)=T_{H_{2}}\left(\mathcal{u}_{i}\right) I_{H_{1}}\left(\mathcal{u}_{i}\right)=I_{H_{2}}\left(\mathcal{u}_{i}\right), F_{H_{1}}\left(\mathcal{u}_{i}\right)=F_{H_{2}}\left(\mathcal{u}_{i}\right), \forall u_{i} \in U$.
iii. $\quad \mathrm{CE}_{\mathrm{NS}}^{\mathrm{w}}\left(\mathrm{H}_{1}, \mathrm{H}_{2}\right)=\mathrm{CE}_{\mathrm{NS}}^{\mathrm{w}}\left(\mathrm{H}_{1^{\prime}}^{\mathrm{c}} \mathrm{H}_{2}^{\mathrm{c}}\right)$
iv. $\mathrm{CE}_{\mathrm{NS}}^{\mathrm{w}}\left(\mathrm{H}_{1}, \mathrm{H}_{2}\right)=\mathrm{CE}_{\mathrm{NS}}^{\mathrm{w}}\left(\mathrm{H}_{2}, \mathrm{H}_{1}\right)$

Proof. (i). For all values of $u_{i} \in U,\left|T_{H_{1}}\left(\mathcal{u}_{i}\right)\right| \geq 0\left|T_{H_{2}}\left(\mathcal{u}_{i}\right)\right| \geq 0,\left|T_{H_{1}}\left(\mathcal{u}_{i}\right)-T_{H_{2}}\left(\mathcal{u}_{i}\right)\right| \geq 0, \sqrt{1+\left|T_{H_{1}}\left(\mathcal{u}_{i}\right)\right|^{2}} \geq 0$, $\sqrt{1+\left|T_{H_{2}}\left(\mathcal{U}_{i}\right)\right|^{2}} \geq 0,\left|\left(1-T_{H_{1}}\left(\mathcal{U}_{i}\right)\right)\right| \geq 0,\left|\left(1-T_{H_{2}}\left(\mathcal{u}_{i}\right)\right)\right| \geq 0,\left|\left(1-T_{H_{1}}\left(\mathcal{U}_{i}\right)\right)-\left(1-T_{H_{2}}\left(\mathcal{U}_{i}\right)\right)\right| \geq 0, \sqrt{1+\left|\left(1-T_{H_{1}}\left(\mathcal{U}_{i}\right)\right)\right|^{2}} \geq 0$, $\sqrt{1+\mid\left(1-\left.T_{H_{2}}\left(\mathcal{U}_{i}\right)\right|^{2}\right.} \geq 0$, then, $\left[\frac{2\left|T_{H_{1}}\left(\mathcal{u}_{i}\right)-T_{H_{2}}\left(\mathcal{u}_{i}\right)\right|}{\sqrt{1+\mid T_{H_{1}}\left(\mathcal{u}_{i}\right)^{2}}+\sqrt{1+\left|T_{H_{2}}\left(\mathcal{u}_{i}\right)\right|^{2}}}+\frac{2\left|\left(1-T_{H_{1}}\left(\mathcal{u}_{i}\right)\right)-\left(1-T_{H_{2}}\left(\mathcal{u}_{i}\right)\right)\right|}{\sqrt{1+\left|\left(1-T_{H_{1}}\left(\mathcal{U}_{i}\right)\right)\right|^{2}}+\sqrt{1+\left|\left(1-T_{H_{2}}\left(\mathcal{u}_{i}\right)\right)\right|^{2}}}\right] \geq 0$. 
Similarly, $\left[\frac{2\left|I_{H_{1}}\left(\mathcal{u}_{i}\right)-I_{H_{2}}\left(\mathcal{u}_{i}\right)\right|}{\sqrt{1+\left|I_{H_{1}}\left(\mathcal{U}_{i}\right)\right|^{2}}+\sqrt{1+\left|I_{H_{2}}(u)\right|^{2}}}+\frac{2\left(1-I_{H_{1}}\left(\mathcal{u}_{i}\right)\right)-\left(1-I_{H_{2}}\left(\mathcal{u}_{i}\right)\right) \mid}{\sqrt{1+\left|\left(1-I_{H_{1}}\left(\mathcal{u}_{i}\right)\right)\right|^{2}}+\sqrt{1+\left|\left(1-I_{H_{2}}\left(\mathcal{u}_{i}\right)\right)\right|^{2}}}\right] \geq 0$, and

$\left[\frac{2\left|F_{H_{1}}\left(\mathcal{u}_{i}\right)-F_{H_{2}}\left(\mathcal{u}_{i}\right)\right|}{\sqrt{1+\left|F_{H_{1}}\left(\mathcal{u}_{i}\right)\right|^{2}}+\sqrt{1+\left|F_{H_{2}}\left(\mathcal{u}_{i}\right)\right|^{2}}}+\frac{2 \mid\left(1-F_{H_{1}}\left(\mathcal{u}_{i}\right)\right)-\left(1-F_{H_{2}}\left(\mathcal{u}_{i}\right) \mid\right.}{\sqrt{1+\left|\left(1-F_{H_{1}}\left(\mathcal{u}_{i}\right)\right)\right|^{2}}+\sqrt{1+\mid\left(1-F_{H_{2}}\left(\mathcal{u}_{i}\right)\right)^{2}}}\right] \geq 0$.

Since $\mathrm{w}_{\mathrm{i}} \in[0,1]$ and $\sum_{i=1}^{n} w_{i}=1$, therefore, $\mathrm{CE}_{\mathrm{NS}}^{\mathrm{w}}\left(\mathrm{H}_{1}, \mathrm{H}_{2}\right) \geq 0$.

Hence complete the proof.

(ii) Since,

$$
\left[\frac{2 \mid T_{H_{1}}\left(\mathcal{u}_{i}\right)-T_{H_{2}}\left(\mathcal{u}_{i} \mid\right.}{\sqrt{1+\left|T_{H_{1}}\left(\mathcal{u}_{i}\right)\right|^{2}}+\sqrt{1+\left|T_{H_{2}}\left(\mathcal{u}_{i}\right)\right|^{2}}}+\frac{2\left|\left(1-T_{H_{1}}\left(\mathcal{u}_{i}\right)\right)-\left(1-T_{H_{2}}\left(\mathcal{u}_{i}\right)\right)\right|}{\sqrt{1+\mid\left(1-T_{H_{1}}\left(\mathcal{u}_{i}\right)\right)^{2}}+\sqrt{1+\mid\left(1-\left.T_{H_{2}}\left(\mathcal{U}_{i}\right)\right|^{2}\right.}}\right]=0, \Leftrightarrow T_{H_{1}}\left(\mathcal{u}_{i}\right)=T_{H_{2}}\left(\mathcal{u}_{i}\right) \quad,
$$

$\left[\frac{2\left|I_{H_{1}}\left(\mathcal{u}_{i}\right)-I_{H_{2}}\left(\mathcal{u}_{i}\right)\right|}{\sqrt{1+\left|I_{H_{1}}\left(\mathcal{u}_{i}\right)\right|^{2}}+\sqrt{1+\left|I_{H_{2}}(u)\right|^{2}}}+\frac{2 \mid\left(1-I_{H_{1}}\left(\mathcal{u}_{i}\right)\right)-\left(1-I_{H_{2}}\left(\mathcal{U}_{i}\right) \mid\right.}{\sqrt{1+\mid\left(1-\left.I_{H_{1}}\left(\mathcal{U}_{i}\right)\right|^{2}\right.}+\sqrt{1+\mid\left(1-\left.I_{H_{2}}\left(\mathcal{u}_{i}\right)\right|^{2}\right.}}\right]=0 \quad, \quad \Leftrightarrow I_{H_{1}}\left(\mathcal{u}_{i}\right)=I_{H_{2}}\left(\mathcal{u}_{i}\right) \quad$, $\left[\frac{2\left|F_{H_{1}}\left(\mathcal{u}_{i}\right)-F_{H_{2}}\left(\mathcal{u}_{i}\right)\right|}{\sqrt{1+\left|F_{H_{1}}\left(\mathcal{u}_{i}\right)\right|^{2}}+\sqrt{1+\left|F_{H_{2}}\left(\mathcal{u}_{i}\right)\right|^{2}}}+\frac{2\left|\left(1-F_{H_{1}}\left(\mathcal{u}_{i}\right)\right)-\left(1-F_{H_{2}}\left(\mathcal{u}_{i}\right)\right)\right|}{\sqrt{1+\mid\left(1-F_{H_{1}}\left(\mathcal{u}_{i}\right)\right)^{2}}+\sqrt{1+\mid\left(1-\left.F_{H_{2}}\left(\mathcal{u}_{i}\right)\right|^{2}\right.}}\right]=0, \quad \Leftrightarrow F_{H_{1}}\left(\mathcal{u}_{i}\right)=F_{H_{2}}\left(\mathcal{u}_{i}\right) \quad$ and $\mathrm{w}_{\mathrm{i}} \in[0,1], \sum_{i=1}^{n} w_{i}=1 \quad, \quad \mathrm{w}_{\mathrm{i}} \geq 0$. Therefore, $\quad \mathrm{CE}_{\mathrm{NS}}^{\mathrm{w}}\left(\mathrm{H}_{1}, \mathrm{H}_{2}\right)=0 \quad$ iff $\quad T_{H_{1}}\left(\mathcal{U}_{i}\right)=T_{H_{2}}\left(\mathcal{U}_{i}\right), \quad I_{H_{1}}\left(\mathcal{U}_{i}\right)=I_{H_{2}}\left(\mathcal{u}_{i}\right)$ ， $F_{H_{1}}\left(u_{i}\right)=F_{H_{2}}\left(u_{i}\right), \forall u_{i} \in U$.

Hence complete the proof.

(iii) Using Definition 5, we obtain the following expression

$$
\begin{aligned}
& \mathrm{CE}_{\mathrm{NS}}^{\mathrm{w}}\left(\mathrm{H}_{1}^{\mathrm{c}}, \mathrm{H}_{2}^{\mathrm{c}}\right)=\frac{1}{2}\left\{\sum _ { i = 1 } ^ { n } w _ { i } \left(\left[\frac{2\left|\left(1-T_{H_{1}}\left(\mathcal{u}_{i}\right)\right)-\left(1-T_{H_{2}}\left(\mathcal{u}_{i}\right)\right)\right|}{\sqrt{1+\mid\left(1-\left.T_{H_{1}}\left(\mathcal{u}_{i}\right)\right|^{2}\right.}+\sqrt{1+\mid\left(1-\left.T_{H_{2}}\left(\mathcal{u}_{i}\right)\right|^{2}\right.}}+\frac{2\left|T_{H_{1}}\left(\mathcal{u}_{i}\right)-T_{H_{2}}\left(\mathcal{u}_{i}\right)\right|}{\sqrt{1+\left|T_{H_{1}}\left(\mathcal{u}_{i}\right)\right|^{2}}+\sqrt{1+\left|T_{H_{2}}\left(\mathcal{u}_{i}\right)\right|^{2}}}\right]+\right.\right. \\
& {\left[\frac{2\left|\left(1-I_{H_{1}}\left(\mathcal{U}_{i}\right)\right)-\left(1-I_{H_{2}}\left(\mathcal{U}_{i}\right)\right)\right|}{\sqrt{1+\left|\left(1-I_{H_{1}}\left(\mathcal{U}_{i}\right)\right)\right|^{2}}+\sqrt{1+\mid\left(1-\left.I_{H_{2}}\left(\mathcal{U}_{i}\right)\right|^{2}\right.}}+\frac{2 \mid I_{H_{1}}\left(\mathcal{U}_{i}\right)-I_{H_{2}}\left(\mathcal{U}_{i} \mid\right.}{\sqrt{1+\left|I_{H_{1}}\left(\mathcal{U}_{i}\right)\right|^{2}}+\sqrt{1+\left|I_{H_{2}}(u)\right|^{2}}}\right]+} \\
& \left.\left.\left[\frac{2 \mid\left(1-F_{H_{1}}\left(u_{i}\right)\right)-\left(1-F_{H_{2}}\left(\mathcal{u}_{i}\right) \mid\right.}{\sqrt{1+\left|\left(1-F_{H_{1}}\left(u_{i}\right)\right)\right|^{2}}+\sqrt{1+\mid\left(1-F_{H_{2}}\left(u_{i}\right)\right)^{2}}}+\frac{2\left|F_{H_{1}}\left(u_{i}\right)-F_{H_{2}}\left(\mathcal{u}_{i}\right)\right|}{\sqrt{1+\left|F_{H_{1}}\left(u_{i}\right)\right|^{2}}+\sqrt{1+\left|F_{H_{2}}\left(\mathcal{u}_{i}\right)\right|^{2}}}\right]\right)\right\} \\
& =\frac{1}{2}\left\{\sum _ { i = 1 } ^ { n } w _ { i } \left(\left[\frac{2 \mid T_{H_{1}}\left(\mathcal{u}_{i}\right)-T_{H_{2}}\left(\mathcal{u}_{i}\right)}{\sqrt{1+\left|T_{H_{1}}\left(\mathcal{u}_{i}\right)\right|^{2}}+\sqrt{1+\left|T_{H_{2}}\left(\mathcal{u}_{i}\right)\right|^{2}}}+\frac{2\left|\left(1-T_{H_{1}}\left(\mathcal{u}_{i}\right)\right)-\left(1-T_{H_{2}}\left(\mathcal{u}_{i}\right)\right)\right|}{\sqrt{1+\left|\left(1-T_{H_{1}}\left(\mathcal{u}_{i}\right)\right)\right|^{2}}+\sqrt{1+\mid\left(1-\left.T_{H_{2}}\left(\mathcal{u}_{i}\right)\right|^{2}\right.}}\right]+\right.\right. \\
& {\left[\frac{2 \mid I_{H_{1}}\left(\mathcal{u}_{i}\right)-I_{H_{2}}\left(\mathcal{u}_{i} \mid\right.}{\sqrt{1+\left|I_{H_{1}}\left(\mathcal{u}_{i}\right)\right|^{2}}+\sqrt{1+\left|I_{H_{2}}(u)\right|^{2}}}+\frac{2 \mid\left(1-I_{H_{1}}\left(\mathcal{u}_{i}\right)\right)-\left(1-I_{H_{2}}\left(\mathcal{u}_{i}\right) \mid\right.}{\sqrt{1+\mid\left(1-\left.I_{H_{1}}\left(\mathcal{U}_{i}\right)\right|^{2}\right.}+\sqrt{1+\mid\left(1-I_{H_{2}}\left(\mathcal{u}_{i}\right)\right)^{2}}}\right]+} \\
& \left.\left[\frac{2\left|F_{H_{1}}\left(\mathcal{u}_{i}\right)-F_{H_{2}}\left(\mathcal{u}_{i}\right)\right|}{\sqrt{1+\left|F_{H_{1}}\left(\mathcal{u}_{i}\right)\right|^{2}}+\sqrt{1+\left|F_{H_{2}}\left(\mathcal{u}_{i}\right)\right|^{2}}}+\frac{2 \mid\left(1-F_{H_{1}}\left(\mathcal{u}_{i}\right)\right)-\left(1-F_{H_{2}}\left(\mathcal{u}_{i}\right) \mid\right.}{\sqrt{1+\left|\left(1-F_{H_{1}}\left(\mathcal{u}_{i}\right)\right)\right|^{2}}+\sqrt{1+\mid\left(1-F_{H_{2}}\left(\mathcal{u}_{i}\right)\right)^{2}}}\right]\right\}=\mathrm{CE}_{N S}^{w}\left(\mathrm{H}_{1}, \mathrm{H}_{2}\right)
\end{aligned}
$$


Therefore, $\mathrm{CE}_{\mathrm{NS}}^{\mathrm{w}}\left(\mathrm{H}_{1}, \mathrm{H}_{2}\right)=\mathrm{CE}_{\mathrm{NS}}^{\mathrm{w}}\left(\mathrm{H}_{1^{\prime}}^{\mathrm{c}} \mathrm{H}_{2}^{\mathrm{c}}\right)$.

Hence complete the proof.

$$
\begin{aligned}
& \left|F_{H_{1}}\left(\mathcal{u}_{i}\right)-F_{H_{2}}\left(\mathcal{u}_{i}\right)\right|=\left|F_{H_{2}}\left(\mathcal{u}_{i}\right)-F_{H_{1}}\left(\mathcal{u}_{i}\right)\right| \quad, \quad\left|\left(1-T_{H_{1}}\left(\mathcal{u}_{i}\right)\right)-\left(1-T_{H_{2}}\left(\mathcal{u}_{i}\right)\right)\right|=\left|\left(1-T_{H_{2}}\left(\mathcal{u}_{i}\right)\right)-\left(1-T_{H_{1}}\left(\mathcal{u}_{i}\right)\right)\right| \text {, } \\
& \left|\left(1-I_{H_{1}}\left(\mathcal{u}_{i}\right)\right)-\left(1-I_{H_{2}}\left(\mathcal{U}_{i}\right)\right)\right|=\left|\left(1-I_{H_{2}}\left(\mathcal{u}_{i}\right)\right)-\left(1-I_{H_{1}}\left(\mathcal{U}_{i}\right)\right)\right|, \quad\left|\left(1-F_{H_{1}}\left(\mathcal{U}_{i}\right)\right)-\left(1-F_{H_{2}}\left(\mathcal{U}_{i}\right)\right)\right|=\left|\left(1-F_{H_{2}}\left(\mathcal{U}_{i}\right)\right)-\left(1-F_{H_{1}}\left(\mathcal{U}_{i}\right)\right)\right| \text {, } \\
& \text { we } \quad \text { obtain } \quad \sqrt{1+\left|T_{H_{1}}\left(\mathcal{u}_{i}\right)\right|^{2}}+\sqrt{1+\left|T_{H_{2}}\left(\mathcal{u}_{i}\right)\right|^{2}}=\sqrt{1+\mid T_{H_{2}}\left(\mathcal{u}_{i}\right)^{2}}+\sqrt{1+\mid T_{H_{1}}\left(\mathcal{u}_{i}\right)^{2}} \\
& \sqrt{1+\left|I_{H_{1}}\left(\mathcal{u}_{i}\right)\right|^{2}}+\sqrt{1+\left|I_{H_{2}}\left(\mathcal{U}_{i}\right)\right|^{2}}=\sqrt{1+\mid I_{H_{2}}\left(\mathcal{U}_{i}\right)^{2}}+\sqrt{1+\mid I_{H_{1}}\left(\mathcal{u}_{i}\right)^{2}} \\
& \sqrt{1+\left|F_{H_{1}}\left(\mathcal{u}_{i}\right)\right|^{2}}+\sqrt{1+\mid F_{H_{2}}\left(\mathcal{u}_{i}\right)^{2}}=\sqrt{1+\mid F_{H_{2}}\left(\left.\mathcal{u}_{i}\right|^{2}\right.}+\sqrt{1+\mid F_{H_{1}}\left(\left.\mathcal{u}_{i}\right|^{2}\right.} \\
& \sqrt{1+\left|\left(1-T_{H_{1}}\left(\mathcal{U}_{i}\right)\right)\right|^{2}}+\sqrt{1+\left|\left(1-T_{H_{2}}\left(\mathcal{U}_{i}\right)\right)\right|^{2}}=\sqrt{1+\left|\left(-T_{H_{2}}\left(\mathcal{U}_{i}\right)\right)\right|^{2}}+\sqrt{1+\left|\left(1-T_{H_{1}}\left(\mathcal{U}_{i}\right)\right)\right|^{2}} \\
& \sqrt{1+\mid\left(1-\left.I_{H_{1}}\left(\mathcal{U}_{i}\right)\right|^{2}\right.}+\sqrt{1+\left|\left(1-I_{H_{2}}\left(\mathcal{U}_{i}\right)\right)\right|^{2}}=\sqrt{1+\mid\left(1-\left.I_{H_{2}}\left(\mathcal{U}_{i}\right)\right|^{2}\right.}+\sqrt{1+\mid\left(1-\left.I_{H_{1}}\left(\mathcal{U}_{i}\right)\right|^{2}\right.} \\
& \sqrt{1+\left|\left(1-F_{H_{1}}\left(\mathcal{u}_{i}\right)\right)\right|^{2}}+\sqrt{1+\left|\left(1-F_{H_{2}}\left(\mathcal{u}_{i}\right)\right)\right|^{2}}=\sqrt{1+\mid\left(1-\left.F_{H_{2}}\left(\mathcal{u}_{i}\right)\right|^{2}\right.}+\sqrt{1+\mid\left(1-\left.F_{H_{1}}\left(u_{i}\right)\right|^{2}\right.} \quad, \quad \forall u_{i} \in U \quad \text { and } \\
& \mathrm{w}_{\mathrm{i}} \in[0,1], \sum_{i=1}^{n} w_{i}=1 \text {. }
\end{aligned}
$$

Therefore, $\mathrm{CE}_{\mathrm{NS}}^{\mathrm{w}}\left(\mathrm{H}_{1}, \mathrm{H}_{2}\right)=\mathrm{CE}_{\mathrm{NS}}^{\mathrm{w}}\left(\mathrm{H}_{2}, \mathrm{H}_{1}\right)$.

Hence complete the proof.

\section{MAGDM Strategy Using Proposed Ns-Cross Entropy Measure under SVNS Environment}

In this section, we develop a new MAGDM strategy using the proposed NS-cross entropy measure.

\section{Description of the MAGDM Problem}

Assume that $A=\left\{A_{1}, A_{2}, A_{3}, \ldots, A_{m}\right\}$ and $G=\left\{G_{1}, G_{2}, G_{3}, \ldots, G_{n}\right\}$ be the discrete set of alternatives and attributes respectively and $W=\left\{w_{1}, w_{2}, w_{3}, \ldots, w_{n}\right\}$ be the weight vector of attributes $G_{j}(j=1,2,3, \ldots, n)$, where $w_{j} \geq 0$ and $\sum_{j=1}^{n} w_{j}=1$. Assume that $E=\left\{E_{1}, E_{2}, E_{3}, \ldots, E_{\rho}\right\}$ be the set of decision-makers who are employed to evaluate the alternatives. The weight vector of the decision-makers $E_{k}(k=1,2,3, \ldots, \rho)$ is $\lambda=\left\{\lambda_{1}, \lambda_{2}, \lambda_{3}, \ldots, \lambda_{\rho}\right\} \quad$ (where, $\lambda_{k} \geq 0$ and $\sum_{k=1}^{\rho} \lambda_{k}=1$ ), which can be determined according to the decision-makers' expertise, judgment quality and domain knowledge.

Now, we describe the steps of the proposed MAGDM strategy (see Figure 1) using NS-cross entropy measure. 


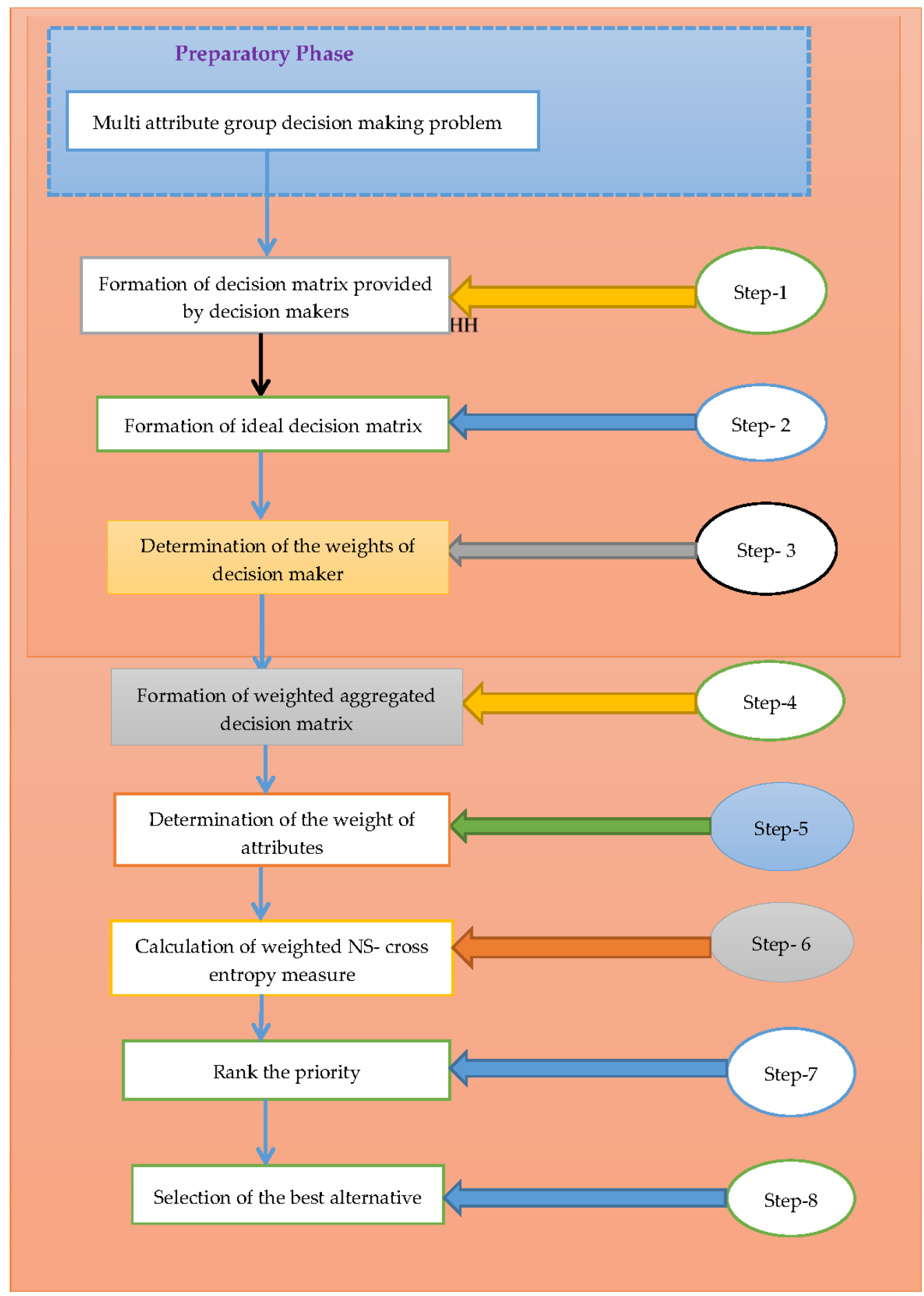

Figure 1. Decision-making procedure of the proposed MAGDM strategy.

\section{MAGDM Strategy Using Ns-Cross Entropy Measure}

\section{Step 1. Formulate the decision matrices}

For MAGDM with SVNSs information, the rating values of the alternatives $A_{i}(i=1,2,3, \ldots, m)$ based on the attribute $G_{j}(j=1,2,3, \ldots, n)$ provided by the $k$-th decision-maker can be expressed in terms of SVNN as $a_{i j}^{k}=<T_{i j}^{k}, I_{i j^{\prime}}^{k} F_{i j}^{k}>(i=1,2,3, \ldots, m ; j=1,2,3, \ldots, n ; k=1,2,3, \ldots, \rho)$. We present these rating values of alternatives provided by the decision-makers in matrix form as follows: 


$$
M^{k}=\left(\begin{array}{ccccc} 
& G_{1} & G_{2} & \ldots & G_{n} \\
A_{1} & \mathrm{a}_{11} & \mathrm{a}_{12} & \cdots & \mathrm{a}_{\mathrm{kn}} \\
A_{2} & \mathrm{a}_{21}^{\mathrm{k}} & \mathrm{a}_{22} & \mathrm{a}^{\mathrm{k}} \\
\cdot & \cdot & \cdots & \cdot & \\
A_{\mathrm{m}} & \mathrm{a}_{\mathrm{m} 1}^{\mathrm{k}} & \mathrm{a}_{\mathrm{m} 2} & \cdots & \mathrm{a}_{\mathrm{mn}}
\end{array}\right)
$$

\section{Step 2. Formulate priori/ideal decision matrix}

In the MAGDM, the a priori decision matrix has been used to select the best alternatives among the set of collected feasible alternatives. In the decision-making situation, we use the following decision matrix as a priori decision matrix.

$$
P=\left(\begin{array}{ccccc} 
& G_{1} & G_{2} & \ldots & G_{n} \\
A_{1} & \mathbf{a}_{11}^{*} & \mathbf{a}_{12}^{*} & \mathbf{a}_{1 \mathrm{n}}^{*} \\
A_{2} & \mathbf{a}_{21}^{*} & \mathbf{a}_{22}^{*} & \mathbf{a}_{2 \mathrm{n}}^{*} \\
\cdot & \cdot & \cdots & \cdot & \\
A_{\mathrm{m}} & \mathbf{a}_{\mathrm{m} 1}^{*} & \mathbf{a}_{\mathrm{m} 2}^{*} & \mathbf{a}_{\mathrm{mn}}^{*}
\end{array}\right)
$$

where, $\left.\mathbf{a}_{\mathrm{ij}}^{*}=<\max _{i}\left(T_{i j}^{k}\right), \min _{i}\left(I_{i j}^{k}\right), \min _{i}\left(F_{i j}^{k}\right)>\right)$ corresponding to benefit attributes and $\mathbf{a}_{\mathrm{ij}}^{*}=<\min _{i}\left(T_{i j}^{k}\right), \max _{i}\left(I_{i j}^{k}\right), \max _{i}\left(F_{i j}^{k}\right)>$ corresponding to cost attributes, and $(i=1,2,3, \ldots, m ; j=1,2,3, \ldots, n ; k$ $=1,2,3, \ldots, \rho)$.

\section{Step 3. Determinate the weights of decision-makers}

To find the decision-makers' weights we introduce a model based on the NS-cross entropy measure. The collective NS-cross entropy measure between $M^{k}$ and $P$ (Ideal matrix) is defined as follows:

$$
C E_{N S}^{c}\left(M^{k}, P\right)=\frac{1}{m} \sum_{i=1}^{m} C E_{N S}\left(\left(M^{k}\left(A_{i}\right), P\left(A_{i}\right)\right)\right.
$$

where, $C E_{N S}\left(M^{k}\left(A_{i}\right), P\left(A_{i}\right)\right)=\sum_{j=1}^{n} C E_{N S}\left(M^{k}\left(A_{i}\left(G_{j}\right)\right), P\left(A_{i}\left(G_{j}\right)\right)\right)$.

Thus, we can introduce the following weight model of the decision-makers:

$$
\lambda_{K}=\frac{\left(1 \div C E_{N S}^{c}\left(M^{k}, P\right)\right)}{\sum_{k=1}^{p}\left(1 \div C E_{N S}^{c}\left(M^{k}, P\right)\right)}
$$

where, $0 \leq \lambda_{K} \leq 1$ and $\sum_{k=1}^{\rho} \lambda_{k}=1$ for $k=1,2,3, \ldots, \rho$.

\section{Step 4. Formulate the weighted aggregated decision matrix}

For obtaining one group decision, we aggregate all the individual decision matrices $\left(\mathrm{M}^{\mathrm{k}}\right)$ to an aggregated decision matrix (M) using single valued neutrosophic weighted averaging (SVNWA) operator ([51]) as follows:

$$
\begin{aligned}
& \mathrm{a}_{\mathrm{ij}}=S V N S W A_{\lambda}\left(\mathbf{a}_{\mathrm{ij}}^{1}, \mathbf{a}_{\mathrm{ij}}^{2}, \mathbf{a}_{\mathrm{ij}}^{3}, \ldots, \mathbf{a}_{\mathrm{ij}}^{\rho}\right)=\left(\lambda_{1} a_{i j}^{1} \oplus \lambda_{2} a_{i j}^{2} \oplus \lambda_{3} a_{i j}^{3} \oplus \ldots \oplus \lambda_{\rho} a_{i j}^{\rho}\right)= \\
& <1-\prod_{k=1}^{\rho}\left(1-T_{i j}^{k}\right)^{\lambda_{k}}, \prod_{k=1}^{\rho}\left(I_{i j}^{k}\right)^{\lambda_{k}}, \prod_{k=1}^{\rho}\left(F_{i j}^{k}\right)^{\lambda_{k}}>
\end{aligned}
$$

Therefore, the aggregated decision matrix is defined as follows: 


$$
M=\left(\begin{array}{lllll} 
& G_{1} & G_{2} & \ldots & G_{n} \\
A_{1} & \mathrm{a}_{11} & \mathrm{a}_{12} \ldots & \mathrm{a}_{1 \mathrm{n}} \\
A_{2} & a_{21} & a_{22} & a_{2 n} \\
\cdot & \cdot & \ldots & . \\
A_{\mathrm{m}} & \mathrm{a}_{\mathrm{m} 1} & \mathrm{a}_{\mathrm{m} 2} \ldots & a_{m n}
\end{array}\right)
$$

where, $\mathrm{a}_{\mathrm{ij}}=<\mathrm{T}_{\mathrm{ij}}, \mathrm{I}_{\mathrm{ij}}, \mathrm{F}_{\mathrm{ij}}>,(i=1,2,3, \ldots, m ; j=1,2,3, \ldots, n ; k=1,2,3, \ldots, \rho)$.

\section{Step 5. Determinate the weight of attributes}

To find the attributes weight we introduce a model based on the NS-cross entropy measure. The collective NS-cross entropy measure between $M$ (Weighted aggregated decision matrix) and $\mathrm{P}$ (Ideal matrix) for each attribute is defined by

$$
C E_{N S}^{j}(M, P)=\frac{1}{m} \sum_{i=1}^{m} C E_{N S}\left(\left(M\left(A_{i}\left(G_{j}\right)\right), P\left(A_{i}\left(G_{j}\right)\right)\right)\right.
$$

where, $i=1,2,3, \ldots, m ; j=1,2,3, \ldots, n$.

Thus, we defined a weight model for attributes as follows:

$$
w_{j}=\frac{\left(1 \div C E_{N S}^{j}(M, P)\right)}{\sum_{J=1}^{n}\left(1 \div C E_{N S}^{j}(M, P)\right)}
$$

where, $0 \leq w_{j} \leq 1$ and $\sum_{j=1}^{n} w_{j}=1$ for $j=1,2,3, \ldots, n$.

\section{Step 6. Calculate the weighted NS-cross entropy measure}

Using Equation (2), we calculate weighted cross entropy value between weighted aggregated matrix and priori matrix. The cross entropy values can be presented in matrix form as follows:

$$
{ }^{N S} M_{C E}^{w}=\left(\begin{array}{l}
\mathrm{CE}_{\mathrm{NS}}^{\mathrm{w}}\left(A_{1}\right) \\
\mathrm{CE}_{\mathrm{NS}}^{\mathrm{w}}\left(A_{2}\right) \\
\cdots \cdots \cdots \cdots \cdots \\
\cdots \cdots \cdots \cdots \cdots \cdots \\
\mathrm{CE}_{\mathrm{NS}}^{\mathrm{w}}\left(A_{\mathrm{m}}\right)
\end{array}\right)
$$

\section{Step 7. Rank the priority}

Smaller value of the cross entropy reflects that an alternative is closer to the ideal alternative. Therefore, the preference priority order of all the alternatives can be determined according to the increasing order of the cross entropy values $\mathrm{CE}_{\mathrm{NS}}^{\mathrm{w}}\left(\mathrm{A}_{\mathrm{i}}\right)(i=1,2,3, \ldots, m)$. Smallest cross entropy value indicates the best alternative and greatest cross entropy value indicates the worst alternative.

\section{Step 8. Select the best alternative}

From the preference rank order (from step 7), we select the best alternative.

\section{Illustrative Example}

In this section, we solve an illustrative example adapted from [12] of MAGDM problems to reflect the feasibility, applicability and efficiency of the proposed strategy under the SVNS environment.

Now, we use the example [12] for cultivation and analysis. A venture capital firm intends to make evaluation and selection of five enterprises with the investment potential:

(1) Automobile company $\left(\mathrm{A}_{1}\right)$

(2) Military manufacturing enterprise $\left(\mathrm{A}_{2}\right)$

(3) TV media company $\left(\mathrm{A}_{3}\right)$ 
(4) Food enterprises $\left(\mathrm{A}_{4}\right)$

(5) Computer software company ( $\left.\mathrm{A}_{5}\right)$

On the basis of four attributes namely:

(1) Social and political factor $\left(\mathrm{G}_{1}\right)$

(2) The environmental factor $\left(\mathrm{G}_{2}\right)$

(3) Investment risk factor (G3)

(4) The enterprise growth factor $\left(\mathrm{G}_{4}\right)$.

The investment firm makes a panel of three decision-makers.

The steps of decision-making strategy (4.1.1.) to rank alternatives are presented as follows:

\section{Step: 1. Formulate the decision matrices}

We represent the rating values of alternatives $A_{i}(i=1,2,3,4,5)$ with respects to the attributes $G_{j}(j=1,2,3,4)$ provided by the decision-makers $E_{k}(k=1,2,3)$ in matrix form as follows:

Decision matrix for $E_{1}$ decision-maker

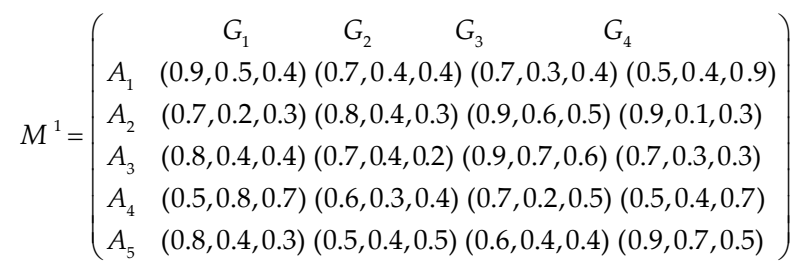

Decision matrix for $E_{2}$ decision-maker

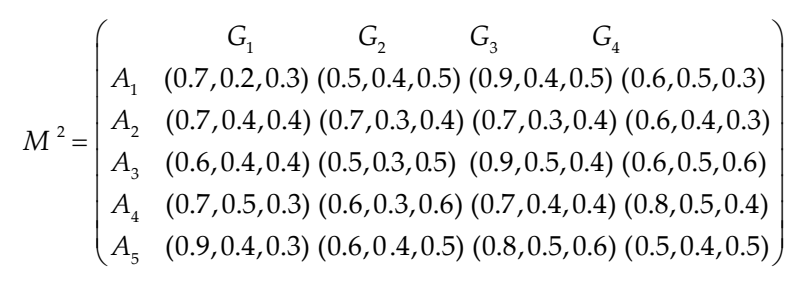

Decision matrix for $E_{3}$ decision-maker

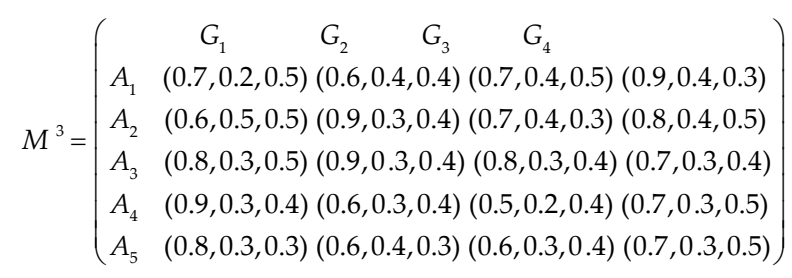

\section{Step: 2. Formulate priori/ideal decision matrix}

A priori/ideal decision matrix Please provide a sharper picture

$$
P=\left(\begin{array}{ccccc} 
& G_{1} & G_{2} & G_{3} & G_{4} \\
A_{1} & (0.9,0.2,0.3) & (0.7,0.4,0.4) & (0.9,0.3,0.4) & (0.9,0.4,0.3) \\
A_{2} & (0.7,0.2,0.3) & (0.9,0.3,0.3) & (0.9,0.3,0.3) & (0.9,0.1,0.3) \\
A_{3} & (0.8,0.3,0.4) & (0.9,0.3,0.2) & (0.9,0.3,0.4) & (0.7,0.3,0.3) \\
A_{4} & (0.9,0.3,0.3) & (0.6,0.3,0.4) & (0.7,0.2,0.4) & (0.7,0.3,0.4) \\
A_{5} & (0.9,0.3,0.3) & (0.6,0.4,0.3) & (0.8,0.3,0.4) & (0.9,0.3,0.5)
\end{array}\right)
$$

\section{Step: 3. Determine the weight of decision-makers}

By using Equations (5) and (6), we determine the weights of the three decision-makers as follows: 


$$
\lambda_{1}=\frac{(1 \div 0.9)}{3.37} \approx 0.33, \lambda_{2}=\frac{(1 \div 1.2)}{3.37} \approx 0.25, \lambda_{1}=\frac{(1 \div .07)}{3.37} \approx 0.42 .
$$

\section{Step: 4. Formulate the weighted aggregated decision matrix}

Using Equation (7) the weighted aggregated decision matrix is presented as follows:

Weighted Aggregated decision matrix

$$
M=\left(\begin{array}{ccccc} 
& G_{1} & G_{2} & G_{3} & G_{4} \\
A_{1} & (0.8,0.3,0.4) & (0.6,0.4,0.4) & (0.8,0.4,0.4) & (0.7,0.4,0.5) \\
A_{2} & (0.7,0.3,0.4) & (0.8,0.3,0.4) & (0.8,0.4,0.4) & (0.8,0.2,0.3) \\
A_{3} & (0.8,0.4,0.4) & (0.8,0.3,0.3) & (0.9,0.5,0.5) & (0.7,0.3,0.4) \\
A_{4} & (0.7,0.5,0.5) & (0.6,0.3,0.4) & (0.6,0.2,0.4) & (0.7,0.4,0.5) \\
A_{5} & (0.8,0.4,0.4) & (0.6,0.4,0.4) & (0.7,0.4,0.4) & (0.8,0.5,0.5)
\end{array}\right)
$$

\section{Step: 5 . Determinate the weight of the attributes}

By using Equations (9) and (10), we determine the weights of the four attribute as follows:

$$
w_{1}=\frac{(1 \div 0.26)}{25} \approx 0.16, w_{2}=\frac{(1 \div 0.11)}{25} \approx 0.37, w_{3}=\frac{(1 \div 0.20)}{25} \approx 0.20, w_{4}=\frac{(1 \div 0.15)}{25} \approx 0.27 \text {. }
$$

\section{Step: 6. Calculate the weighted SVNS cross entropy matrix}

Using Equation (2) and weights of attributes, we calculate the weighted NS-cross entropy values between ideal matrix and weighted aggregated decision matrix.

$$
{ }^{N S} M_{C E}^{w}=\left(\begin{array}{l}
0.195 \\
0.198 \\
0.168 \\
0.151 \\
0.184
\end{array}\right)
$$

\section{Step: 7. Rank the priority}

The cross entropy values of alternatives are arranged in increasing order as follows:

$$
0.151<0.168<0.184<0.195<0.198 .
$$

Alternatives are then preference ranked as follows:

$$
\mathrm{A}_{4}>\mathrm{A}_{3}>\mathrm{A}_{5}>\mathrm{A}_{1}>\mathrm{A}_{2} .
$$

\section{Step: 8 . Select the best alternative}

From step 7, we identify $A_{4}$ is the best alternative. Hence, Food enterprises $\left(A_{4}\right)$ is the best alternative for investment.

In Figure 2, we draw a bar diagram to represent the cross entropy values of alternatives which shows that $\mathrm{A}_{4}$ is the best alternative according our proposed strategy.

In Figure 3, we represent the relation between cross entropy values and acceptance values of alternatives. The range of acceptance level for five alternatives is taken by five points. The high acceptance level of alternatives indicates the best alternative for acceptance and low acceptance level of alternative indicates the poor acceptance alternative.

We see from Figure 3 that alternative $\mathrm{A}_{4}$ has the smallest cross entropy value and the highest acceptance level. Therefore $A_{4}$ is the best alternative for acceptance. Figure 3 indicates that alternative $A_{2}$ has highest cross entropy value and lowest acceptance value that means $A_{2}$ is the worst alternative. Finally, we conclude that the relation between cross entropy values and acceptance value of alternatives is opposite in nature. 


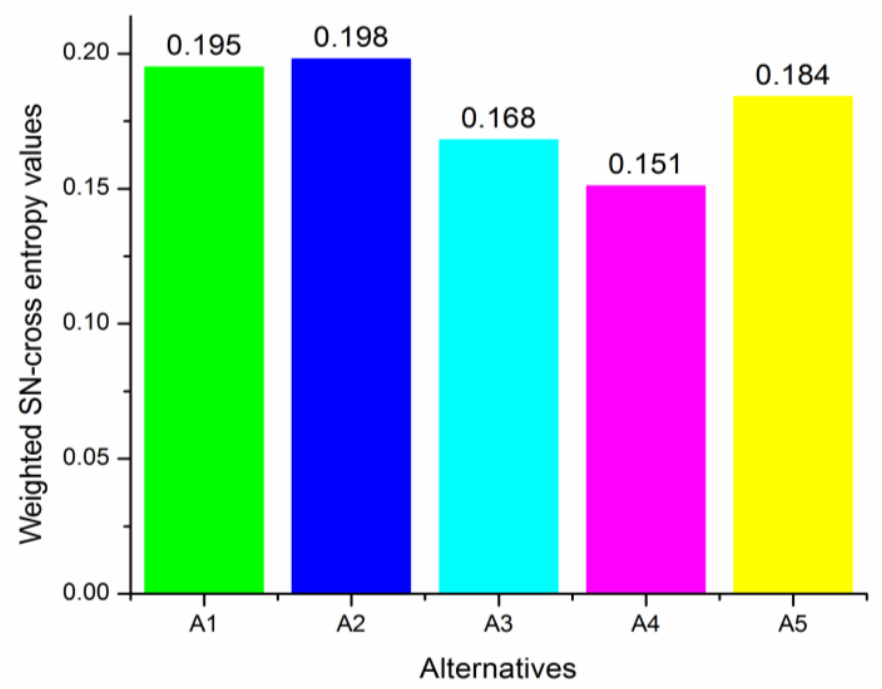

Figure 2. Bar diagram of alternatives versus weighted NS-cross entropy values of alternatives.

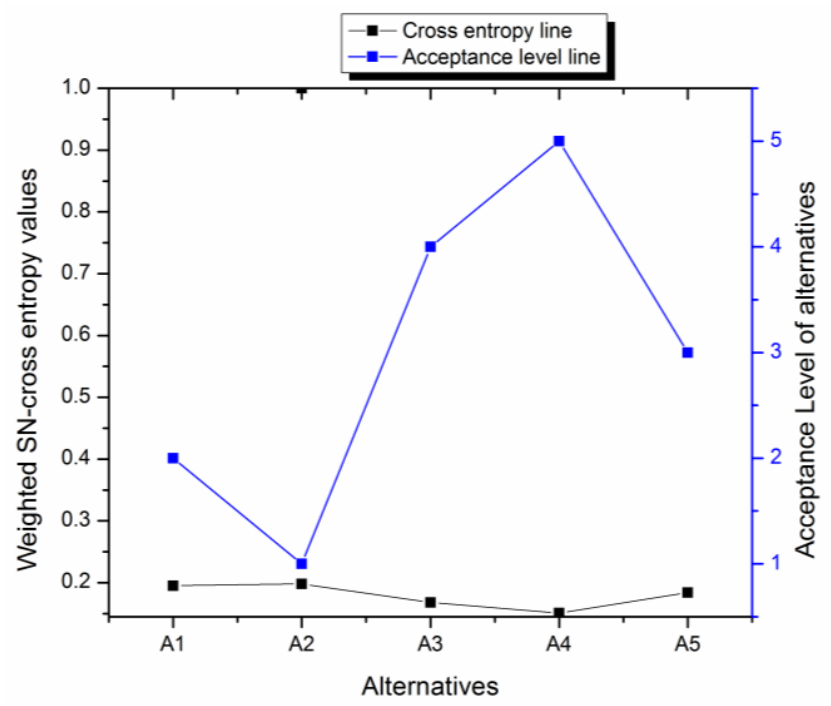

Figure 3. Relation between weighted NS-cross entropy values and acceptance level line of alternatives.

\section{Comparative Study and Discussion}

In literature only two MADM strategies $[144,145]$ have been proposed. No MADGM strategy is available. So the proposed MAGDM is novel and non-comparable with the existing cross entropy under SVNS for numerical example.

i. The MADM strategies $[144,145]$ are not applicable for MAGDM problems. The proposed MAGDM strategy is free from such drawbacks.

ii. Ye [144] proposed cross entropy that does not satisfy the symmetrical property straightforward and is undefined for some situations but the proposed strategy satisfies symmetric property and is free from undefined phenomenon.

iii. The strategies $[144,145]$ cannot deal with the unknown weight of the attributes whereas the proposed MADGM strategy can deal with the unknown weight of the attributes

iv. The strategies [144,145] are not suitable for dealing with the unknown weight of decision-makers, whereas the essence of the proposed NS-cross entropy-based MAGDM is that it is capable of dealing with the unknown weight of the decision-makers. 


\section{Conclusions}

In this paper, we have defined a novel cross entropy measure in SVNS environment. The proposed cross entropy measure in SVNS environment is free from the drawbacks of asymmetrical behavior and undefined phenomena. It is capable of dealing with the unknown weight of attributes and the unknown weight of decision-makers. We have proved the basic properties of the NS-cross entropy measure. We also defined weighted NS-cross entropy measure and proved its basic properties. Based on the weighted NS-cross entropy measure, we have developed a novel MAGDM strategy to solve neutrosophic multi-attribute group decision-making problems. We have at first proposed a novel MAGDM strategy based on NS-cross entropy measure with technique to determine the unknown weight of attributes and the unknown weight of decision-makers. Other existing cross entropy measures [144,145] can deal only with the MADM problem with single decision-maker and known weight of the attributes. So in general, our proposed NS-cross entropy-based MAGDM strategy is not comparable with the existing cross-entropy-based MADM strategies [144,145] under the single-valued neutrosophic environment. Finally, we solve a MAGDM problem to show the feasibility, applicability and efficiency of the proposed MAGDM strategy. The proposed NS-cross entropy-based MAGDM can be applied in teacher selection, pattern recognition, weaver selection, medical treatment selection options, and other practical problems. In future study, the proposed NS-cross entropy-based MAGDM strategy can be also extended to the interval neutrosophic set environment.

Acknowledgments: The authors are very grateful to the anonymous reviewers for their insightful and constructive comments and suggestions that have led to an improved version of this paper.

Author Contributions: Surapati Pramanik conceived and designed the problem; Surapati Pramanik and Shyamal Dalapati solved the problem; Surapati Pramanik, Shariful Alam, Florentin Smarandache and Tapan Kumar Roy analyzed the results; Surapati Pramanik and Shyamal Dalapati wrote the paper.

Conflicts of Interest: The authors declare no conflicts of interest.

\section{References}

1. Zadeh, L.A. Fuzzy sets. Inf. Control 1965, 8, 338-356.

2. Bellman, R.; Zadeh, L.A. Decision-making in A fuzzy environment. Manag. Sci. 1970, 17, 141-164.

3. Atanassov, K.T. Intuitionistic fuzzy sets. Fuzzy Sets Syst. 1986, 20, 87-96.

4. Pramanik, S.; Mukhopadhyaya, D. Grey relational analysis-based intuitionistic fuzzy multi-criteria group decision-making approach for teacher selection in higher education. Int. J. Comput. Appl. 2011, 34, 21-29, doi:10.5120/4138-5985.

5. Mondal, K.; Pramanik, S. Intuitionistic fuzzy multi criteria group decision making approach to quality-brick selection problem. J. Appl. Quant. Methods 2014, 9, 35-50.

6. Dey, P.P.; Pramanik, S.; Giri, B.C. Multi-criteria group decision making in intuitionistic fuzzy environment based on grey relational analysis for weaver selection in Khadi institution. J. Appl. Quant. Methods 2015, 10, $1-14$.

7. Ye, J. Multicriteria fuzzy decision-making method based on the intuitionistic fuzzy cross-entropy. In Proceedings of the International Conference on Intelligent Human-Machine Systems and Cybernetics, Hangzhou, China, 26-27 August 2009; Volume 1, pp. 59-61.

8. Chen, S.M.; Chang, C.H. A novel similarity measure between Atanassov's intuitionistic fuzzy sets based on transformation techniques with applications to pattern recognition. Inf. Sci. 2015, 291, 96-114.

9. Chen, S.M.; Cheng, S.H.; Chiou, C.H. Fuzzy multi-attribute group decision making based on intuitionistic fuzzy sets and evidential reasoning methodology. Inf. Fusion 2016, 27, 215-227.

10. Wang, J.Q.; Han, Z.Q.; Zhang, H.Y. Multi-criteria group decision making method based on intuitionistic interval fuzzy information. Group Decis. Negot. 2014, 23, 715-733.

11. Yue, Z.L. TOPSIS-based group decision-making methodology in intuitionistic fuzzy setting. Inf. Sci. 2014, 277, 141-153.

12. He, X.; Liu, W.F. An intuitionistic fuzzy multi-attribute decision-making method with preference on alternatives. Oper. Res. Manag. Sci. 2013, 22, 36-40. 
13. Zadeh, L.A. Probability Measures of Fuzzy Events. J. Math. Anal. Appl. 1968, 23, 421-427.

14. Burillo, P.; Bustince, H. Entropy on intuitionistic fuzzy sets and on interval-valued fuzzy sets. Fuzzy Sets Systs. 1996, 78, 305-316.

15. Szmidt, E.; Kacprzyk, J. Entropy for intuitionistic fuzzy sets. Fuzzy Sets Syst. 2001, 118, 467-477.

16. Wei, C.P.; Wang, P.; Zhang, Y.Z. Entropy, similarity measure of interval-valued intuitionistic fuzzy sets and their applications. Inf. Sci. 2011, 181, 4273-4286.

17. Li, X.Y. Interval-valued intuitionistic fuzzy continuous cross entropy and its application in multi-attribute decision-making. Comput. Eng. Appl. 2013, 49, 234-237.

18. Shang, X.G.; Jiang, W.S. A note on fuzzy information measures. Pattern Recognit. Lett. 1997, 18, 425-432.

19. Vlachos, I.K.; Sergiadis, G.D. Intuitionistic fuzzy information applications to pattern recognition. Pattern Recognit. Lett. 2007, 28, 197-206.

20. Ye, J. Fuzzy cross entropy of interval-valued intuitionistic fuzzy sets and its optimal decision-making method based on the weights of alternatives. Expert Syst. Appl. 2011, 38, 6179-6183.

21. Xia, M.M.; Xu, Z.S. Entropy/cross entropy-based group decision making under intuitionistic fuzzy environment. Inf. Fusion 2012, 13, 31-47.

22. Tong, X.; Yu, L. A novel MADM approach based on fuzzy cross entropy with interval-valued intuitionistic fuzzy sets. Math. Probl. Eng. 2015, 2015, 965040, doi:10.1155/2015/965040.

23. Smarandache, F. Neutrosophy, Neutrosophic Probability, Set, and Logic, 4th ed.; American Research Press: Rehoboth, DE, USA, 1998.

24. Wang, H.; Smarandache, F.; Zhang, Y.Q.; Sunderraman, R. Single valued neutrosophic sets. Multispace Multistruct. 2010, 4, 410-413.

25. Pramanik, S.; Biswas, P.; Giri, B.C. Hybrid vector similarity measures and their applications to multi-attribute decision making under neutrosophic environment. Neural Comput. Appl. 2017, 28, 1163-1176, doi:10.1007/s00521-015-2125-3.

26. Biswas, P.; Pramanik, S.; Giri, B.C. Entropy based grey relational analysis method for multi-attribute decision making under single valued neutrosophic assessments. Neutrosoph. Sets Syst. 2014, 2, 102-110.

27. Biswas, P.; Pramanik, S.; Giri, B.C. A new methodology for neutrosophic multi-attribute decision making with unknown weight information. Neutrosoph. Sets Syst. 2014, 3, 42-52.

28. Biswas, P.; Pramanik, S.; Giri, B.C. TOPSIS method for multi-attribute group decision-making under single valued neutrosophic environment. Neural Comput. Appl. 2016, 27, 727-737, doi:10.1007/s00521-015-1891-2.

29. Biswas, P.; Pramanik, S.; Giri, B.C. Aggregation of triangular fuzzy neutrosophic set information and its application to multi-attribute decision making. Neutrosoph. Sets Syst. 2016, 12, 20-40.

30. Biswas, P.; Pramanik, S.; Giri, B.C. Value and ambiguity index based ranking method of single-valued trapezoidal neutrosophic numbers and its application to multi-attribute decision making. Neutrosoph. Sets Syst. 2016, 12, 127-138.

31. Biswas, P.; Pramanik, S.; Giri, B.C. Multi-attribute group decision making based on expected value of neutrosophic trapezoidal numbers. In New Trends in Neutrosophic Theory and Applications; Smarandache, F., Pramanik, S., Eds.; Pons Editions: Brussels, Belgium; Volume II, in press.

32. Biswas, P.; Pramanik, S.; Giri, B.C. Non-linear programming approach for single-valued neutrosophic TOPSIS method. New Math. Nat. Comput. 2017, in press.

33. Deli. I.; Subas, Y. A ranking method of single valued neutrosophic numbers and its applications to multi-attribute decision making problems. Int. J. Mach. Learn. Cybern. 2017, 8, 1309-1322, doi:10.1007/s13042016-0505-3.

34. Ji, P.; Wang, J.Q.; Zhang, H.Y. Frank prioritized Bonferroni mean operator with single-valued neutrosophic sets and its application in selecting third-party logistics providers. Neural Comput. Appl. 2016, doi:10.1007/s00521-016-2660-6.

35. Kharal, A. A neutrosophic multi-criteria decision making method. New Math. Nat. Comput. 2014, 10, 143162.

36. Liang, R.X.; Wang, J.Q.; Li, L. Multi-criteria group decision making method based on interdependent inputs of single valued trapezoidal neutrosophic information. Neural Comput. Appl. 2016, 1-20, doi:10.1007/s00521-016-2672-2.

37. Liang, R.X.; Wang, J.Q.; Zhang, H.Y. A multi-criteria decision-making method based on single-valued trapezoidal neutrosophic preference relations with complete weight information. Neural Comput. Appl. 2017, 1-16, doi:10.1007/s00521-017-2925-8. 
38. Liu, P.; Chu, Y.; Li, Y.; Chen, Y. Some generalized neutrosophic number Hamacher aggregation operators and their application to group decision making. Int. J. Fuzzy Syst. 2014, 16, 242-255.

39. Liu, P.D.; Li, H.G. Multiple attribute decision-making method based on some normal neutrosophic Bonferroni mean operators. Neural Comput. Appl. 2017, 28, 179-194.

40. Liu, P.; Wang, Y. Multiple attribute decision-making method based on single-valued neutrosophic normalized weighted Bonferroni mean. Neural Comput. Appl. 2014, 25, 2001-2010.

41. Peng, J.J.; Wang, J.Q.; Wang, J.; Zhang, H.Y.; Chen, X.H. Simplified neutrosophic sets and their applications in multi-criteria group decision-making problems. Int. J. Syst. Sci. 2016, 47, 2342-2358.

42. Peng, J.; Wang, J.; Zhang, H.; Chen, X. An outranking approach for multi-criteria decision-making problems with simplified neutrosophic sets. Appl. Soft Comput. 2014, 25, 336-346.

43. Zavadskas, E.K.; Baušys, R.; Lazauskas, M. Sustainable assessment of alternative sites for the construction of a waste incineration plant by applying WASPAS method with single-valued neutrosophic set. Sustainability 2015, 7, 15923-15936.

44. Pramanik, S.; Dalapati, S.; Roy, T.K. Logistics center location selection approach based on neutrosophic multi-criteria decision making. In New Trends in Neutrosophic Theory and Applications; Smarandache, F., Pramanik, S., Eds.; Pons Editions: Brussels, Belgium, 2016; Volume 1, pp. 161-174.

45. Sahin, R.; Karabacak, M. A multi attribute decision making method based on inclusion measure for interval neutrosophic sets. Int. J. Eng. Appl. Sci. 2014, 2, 13-15.

46. Sahin, R.; Kucuk, A. Subsethood measure for single valued neutrosophic sets. J. Intell. Fuzzy Syst. 2015, 29, 525-530, doi:10.3233/IFS-141304.

47. Sahin, R.; Liu, P. Maximizing deviation method for neutrosophic multiple attribute decision making with incomplete weight information. Neural Comput. Appl. 2016, 27, 2017-2029.

48. Sodenkamp, M. Models, Strategies and Applications of Group Multiple-Criteria Decision Analysis in Complex and Uncertain Systems. PhD Dissertation, University of Paderborn, Paderborn, Germany, 2013.

49. Ye, J. Multicriteria decision-making method using the correlation coefficient under single-valued neutrosophic environment. Int. J. Gen. Syst. 2013, 42, 386-394.

50. Jiang, W.; Shou, Y. A Novel single-valued neutrosophic set similarity measure and its application in multi criteria decision-making. Symmetry 2017, 9, 127, doi:10.3390/sym9080127.

51. Ye, J. A multi criteria decision-making method using aggregation operators for simplified neutrosophic sets. J. Intell. Fuzzy Syst. 2014, 26, 2459-2466.

52. Xu, D.S.; Wei, C.; Wei, G.W. TODIM method for single-valued neutrosophic multiple attribute decision making. Information 2017, 8, 125.

53. Ye, J. Bidirectional projection method for multiple attribute group decision making with neutrosophic number. Neural Comput. Appl. 2017, 28, 1021-1029, doi:10.1007/s00521-015-2123-5.

54. Ye, J. Projection and bidirectional projection measures of single valued neutrosophic sets and their decision-Making method for mechanical design scheme. J. Exp. Theor. Artif. Intell. 2017, 29, 731-740, doi:10.1080/0952813X.2016.1259263.

55. Pramanik, S.; Roy, T.K. Neutrosophic game theoretic approach to Indo-Pak conflict over Jammu-Kashmir. Neutrosoph. Sets Syst. 2014, 2, 82-101.

56. Mondal, K.; Pramanik, S. Multi-criteria group decision making approach for teacher recruitment in higher education under simplified Neutrosophic environment. Neutrosoph. Sets Syst. 2014, 6, 28-34.

57. Mondal, K.; Pramanik, S. Neutrosophic decision making model of school choice. Neutrosoph. Sets Syst. 2015, 7, 62-68.

58. Cheng, H.D.; Guo, Y. A new neutrosophic approach to image thresholding. New Math. Nat. Comput. 2008, 4, 291-308.

59. Guo, Y.; Cheng, H.D. New neutrosophic approach to image segmentation. Pattern Recognit. 2009, 42, 587595.

60. Guo, Y.; Sengur, A.; Ye, J. A novel image thresholding algorithm based on neutrosophic similarity score. Measurement 2014, 58, 175-186.

61. Ye, J. Single valued neutrosophic minimum spanning tree and its clustering method. J. Intell. Syst. 2014, 23, 311-324.

62. Ye, J. Clustering strategies using distance-based similarity measures of single-valued neutrosophic sets. J. Intell. Syst. 2014, 23, 379-389. 
63. Mondal, K.; Pramanik, S. A study on problems of Hijras in West Bengal based on neutrosophic cognitive maps. Neutrosoph. Sets Syst. 2014, 5, 21-26.

64. Pramanik, S.; Chakrabarti. S. A study on problems of construction workers in West Bengal based on neutrosophic cognitive maps. Int. J. Innov. Res. Sci. Eng. Technol. 2013, 2, 6387-6394.

65. Maji, P.K. Neutrosophic soft set. Ann. Fuzzy Math. Inform. 2012, 5, 157-168.

66. Maji, P.K. Neutrosophic soft set approach to a decision-making problem. Ann. Fuzzy Math. Inform. 2013, 3 , 313-319.

67. Sahin, R.; Kucuk, A. Generalized neutrosophic soft set and its integration to decision-making problem. Appl. Math. Inf. Sci. 2014, 8, 2751-2759.

68. Dey, P.P.; Pramanik, S.; Giri, B.C. Neutrosophic soft multi-attribute decision making based on grey relational projection method. Neutrosoph. Sets Syst. 2016, 11, 98-106.

69. Dey, P.P.; Pramanik, S.; Giri, B.C. Neutrosophic soft multi-attribute group decision making based on grey relational analysis method. J. New Results Sci. 2016, 10, 25-37.

70. Dey, P.P.; Pramanik, S.; Giri, B.C. Generalized neutrosophic soft multi-attribute group decision making based on TOPSIS. Crit. Rev. 2015, 11, 41-55.

71. Pramanik, S.; Dalapati, S. GRA based multi criteria decision making in generalized neutrosophic soft set environment, Glob. J. Eng. Sci. Res. Manag. 2016, 3, 153-169.

72. Das, S.; Kumar, S.; Kar, S.; Pal, T. Group decision making using neutrosophic soft matrix: An algorithmic approach. J. King Saud Univ. Comput. Inf. Sci. 2017, doi:10.1016/j.jksuci.2017.05.001.

73. Şahin, M.; Alkhazaleh, S.; Uluçay, V. Neutrosophic soft expert sets. Appl. Math. 2015, 6, 116-127.

74. Pramanik, S.; Dey, P.P.; Giri, B.C. TOPSIS for single valued neutrosophic soft expert set based multi-attribute decision making problems. Neutrosoph. Sets Syst. 2015, 10, 88-95.

75. Broumi, S.; Smarandache, F. Single valued neutrosophic soft expert sets and their application in decision making. J. New Theory 2015, 3, 67-88.

76. Ali, M.; Smarandache, F. Complex neutrosophic set. Neural Comput. Appl. 2017, 28, 1817-1831, doi:10.1007/s00521-015-2154-y.

77. Broumi, S.; Smarandache, F.; Dhar, M. Rough neutrosophic sets. Ital. J. Pure Appl. Math. 2014, 32, 493-502.

78. Broumi, S.; Smarandache, F.; Dhar, M. Rough neutrosophic sets. Neutrosoph. Sets Syst. 2014, 3, 60-66.

79. Yang, H.L.; Zhang, C.L.; Guo, Z.L.; Liu, Y.L.; Liao, X. A hybrid model of single valued neutrosophic sets and rough sets: Single valued neutrosophic rough set model. Soft Comput. 2016, 21, 6253-6267, doi:10.1007/s00500-016-2356-y.

80. Mondal, K.; Pramanik, S. Rough neutrosophic multi-attribute decision-making based on grey relational analysis. Neutrosoph. Sets Syst. 2015, 7, 8-17.

81. Mondal, K.; Pramanik, S. Rough neutrosophic multi-attribute decision-making based on rough accuracy score function. Neutrosoph. Sets Syst. 2015, 8, 14-21.

82. Mondal, K.; Pramanik, S.; Smarandache, F. Several trigonometric Hamming similarity measures of rough neutrosophic sets and their applications in decision making. In New Trends in Neutrosophic Theory and Applications; Smarandache, F., Pramanik, S., Eds.; Pons Editions: Brussels, Belgium, 2016; Volume 1, pp. $93-103$.

83. Mondal, K.; Pramanik, S.; Smarandache, F. Multi-attribute decision making based on rough neutrosophic variational coefficient similarity measure. Neutrosoph. Sets Syst. 2016, 13, 3-17.

84. Mondal, K.; Pramanik, S.; Smarandache, F. Rough neutrosophic TOPSIS for multi-attribute group decision making. Neutr. Sets Syst. 2016, 13, 105-117.

85. Pramanik, S.; Roy, R.; Roy, T.K.; Smarandache, F. Multi criteria decision making using correlation coefficient under rough neutrosophic environment. Neutrosoph. Sets Syst. 2017, 17, 29-36.

86. Pramanik, S.; Roy, R.; Roy, T.K. Multi criteria decision making based on projection and bidirectional projection measures of rough neutrosophic sets. In New Trends in Neutrosophic Theory and Applications; Smarandache, F., Pramanik, S., Eds.; Pons Editions: Brussels, Belgium, 2017; Volume II.

87. Mondal, K.; Pramanik, S. Tri-complex rough neutrosophic similarity measure and its application in multi-attribute decision making. Crit. Rev. 2015, 11, 26-40.

88. Mondal, K.; Pramanik, S.; Smarandache, F. Rough neutrosophic hyper-complex set and its application to multi-attribute decision making. Crit. Rev. 2016, 13, 111-126.

89. Wang, J.Q.; Li, X.E. TODIM method with multi-valued neutrosophic sets. Control Decis. 2015, 30, 11391142. 
90. Peng, J.J.; Wang, J.Q.; Wu, X.H.; Wang, J.; Chen, X.H. Multi-valued neutrosophic sets and power aggregation operators with their applications in multi-criteria group decision-making problems. Int. J. Comput. Intell. Syst. 2015, 8, 345-363.

91. Peng, J.J.; Wang, J. Multi-valued neutrosophic sets and its application in multi-criteria decision-making problems. Neutrosoph. Sets Syst. 2015, 10, 3-17.

92. Ye, J. Multiple-attribute decision-making method under a single-valued neutrosophic hesitant fuzzy environment. J. Intell. Syst. 2015, 24, 23-36.

93. Sahin, R.; Liu, P. Correlation coefficient of single-valued neutrosophic hesitant fuzzy sets and its applications in decision making. Neural Comput Appl. 2017, 28, 1387-1395, doi:10.1007/s00521-015-2163-x.

94. Liu, P.; Zhang, L. The extended VIKOR method for multiple criteria decision making problem based on neutrosophic hesitant fuzzy set. arXiv 2015, arXiv:1512.0139.

95. Biswas, P.; Pramanik, S.; Giri, B.C. Some distance measures of single valued neutrosophic hesitant fuzzy sets and their applications to multiple attribute decision making. In New Trends in Neutrosophic Theory and Applications; Smarandache, F., Pramanik, S., Eds.; Pons Editions: Brussels, Belgium, 2016; pp. 55-63.

96. Biswas, P.; Pramanik, S.; Giri, B.C. GRA method of multiple attribute decision making with single valued neutrosophic hesitant fuzzy set information. In New Trends in Neutrosophic Theory and Applications; Smarandache, F., Pramanik, S., Eds.; Pons Editions: Brussels, Belgium, 2016; pp. 55-63.

97. Sahin, R.; Liu, P. Distance and similarity measure for multiple attribute with single-valued neutrosophic hesitant fuzzy information. In New Trends in Neutrosophic Theory and Applications; Smarandache, F., Pramanik, S., Eds.; Pons Editions: Brussels, Belgium, 2016; pp. 35-54.

98. Deli, I.; Ali, M.; Smarandache, F. Bipolar neutrosophic sets and their applications based on multi criteria decision making problems. In Proceedings of the 2015 International Conference on Advanced Mechatronic Systems (ICAMechS), Beijing, China, 22-24 August 2015; pp. 249-254, doi:10.1109/ICAMechS.2015.7287068.

99. Dey, P.P.; Pramanik, S.; Giri, B.C. TOPSIS for solving multi-attribute decision making problems under bi-polar neutrosophic environment. In New Trends in Neutrosophic Theory and Applications; Smarandache, F., Pramanik, S., Eds.; Pons Editions: Brussels, Belgium, 2016; pp. 65-77.

100. Pramanik, S.; Dey, P.P.; Giri, B.C.; Smarandache, F. Bipolar neutrosophic projection based models for solving multi-attribute decision making problems. Neutrosoph. Sets Syst. 2017, 15, 70-79.

101. Uluçay, V.; Deli, I.; Şahin, M. Similarity measures of bipolar neutrosophic sets and their application to multiple criteria decision making. Neural Comput. Appl. 2016, 1-10, doi:10.1007/s00521-016-2479-1.

102. Sahin. M.; Deli, I.; Ulucay, V. Jaccard vector similarity measure of bipolar neutrosophic set based on multi-criteria decision making. In Proceedings of the International Conference on Natural Science and Engineering (ICNASE'16), Kilis, Turkey, 19-20 March 2016.

103. Deli, I.; Subas, Y.A. Multiple criteria decision making method on single valued bipolar neutrosophic set based on correlation coefficient similarity measure. In Proceedings of the International Conference on Mathematics and Mathematics Education (ICMME 2016), Elazg, Turkey, 12-14 May 2016.

104. Ali, M.; Deli, I.; Smarandache, F. The theory of neutrosophic cubic sets and their applications in pattern recognition. J. Intell. Fuzzy Syst. 2016, 30, 1957-1963.

105. Jun, Y.B.; Smarandache, F.; Kim, C.S. Neutrosophic cubic sets. New Math. Nat. Comput. 2017, 13, 41-54.

106. Banerjee, D.; Giri, B.C.; Pramanik, S.; Smarandache, F. GRA for multi attribute decision making in neutrosophic cubic set environment. Neutrosoph. Sets Syst. 2017, 15, 60-69.

107. Pramanik, S.; Dalapati, S.; Alam, S.; Roy, T.K. NC-TODIM-based MAGDM under a neutrosophic cubic set environment. Information 2017, 8, 149, doi:10.3390/info8040149.

108. Pramanik, S.; Dalapati, S.; Alam, S.; Roy, T.K.; Smarandache, F. Neutrosophic cubic MCGDM method based on similarity measure. Neutrosoph. Sets Syst. 2017, 16, 44-56.

109. Lu, Z.; Ye, J. Cosine measures of neutrosophic cubic sets for multiple attribute decision-making. Symmetry 2017, 9, 121.

110. Pramanik, S.; Dey, P.P.; Giri, B.C.; Smarandache, F. An Extended TOPSIS for Multi-Attribute Decision Making Problems with Neutrosophic Cubic Information. Neutrosoph. Sets Syst. 2017, 17, 20-28.

111. Zhan, J.; Khan, M.; Gulistan, M. Applications of neutrosophic cubic sets in multi-criteria decision-making. Int. J. Uncertain. Quantif. 2017, 7, 377-394.

112. Ye, J. Linguistic neutrosophic cubic numbers and their multiple attribute decision-making method. Information 2017, 8, 110. 
113. Pramanik, S.; Dalapati, S.; Alam, S.; Roy, T.K. TODIM method for group decision making under bipolar neutrosophic set environment. In New Trends in Neutrosophic Theory and Applications; Smarandache, F., Pramanik, S., Eds.; Pons Editions: Brussels, Belgium, 2017; Volume II.

114. Chinnadurai, V.; Swaminathan, A.; Anu, B. Some properties of neutrosophic cubic soft set. Int. J. Comput. Res. Dev. 2016, 1, 113-119.

115. Pramanik, S.; Dalapati, S.; Alam, S.; Roy, T.K. Some operations and properties of neutrosophic cubic soft set. Glob. J. Res. Rev. 2017, 4, 1-8, doi:10.21767/2393-8854.100014.

116. Wang, H.; Smarandache, F.; Zhang, Y.Q.; Sunderraman, R. Interval Neutrosophic Sets and Logic: Theory and Applications in Computing; Hexis: Phoenix, AZ, USA, 2005.

117. Deli, I. Interval-valued neutrosophic soft sets and its decision making. Int. J. Mach. Learn. Cybern. 2017, 8, 665, doi:10.1007/s13042-015-0461-3.

118. Ali, M.; Dat, L.Q.; Son, L.H.; Smarandache, F. Interval complex neutrosophic set: Formulation and applications in decision-making. Int. J. Fuzzy Syst. 2017, 1-14, doi:10.1007/s40815-017-0380-4.

119. Broumi, S.; Smarandache, F. Interval neutrosophic rough set. Neutrosoph. Sets Syst. 2015, 7, 23-31, doi:10.5281/zenodo.571414.

120. Pramanik, S.; Mondal, K. Interval neutrosophic multi-attribute decision-making based on grey relational analysis. Neutrosoph. Sets Syst. 2015, 9, 13-22, doi:10.5281/zenodo.571413.

121. Mondal, K.; Pramanik, S. Decision making based on some similarity measures under interval rough neutrosophic environment. Neutrosoph. Sets Syst. 2015, 10, 46-57, doi:10.5281/zenodo.571358.

122. Ye, J. Correlation coefficients of interval neutrosophic hesitant fuzzy sets and its application in a multiple attribute decision making method. Informatica 2016, 27, 179-202.

123. Biswas, P.; Pramanik, S.; Giri, B.C. Cosine similarity measure based multi-attribute decision-making with trapezoidal fuzzy neutrosophic numbers. Neutrosoph. Sets Syst. 2015, 8, 47-57.

124. Ye, J. Trapezoidal neutrosophic set and its application to multiple attribute decision-making. Neural Comput. Appl. 2015, 26, 1157-1166.

125. Liu, P.D.; Teng, F. Multiple attribute decision making method based on normal neutrosophic generalized weighted power averaging operator. Int. J. Mach. Learn. Cybern. 2015, 1-13, doi:10.1007/s13042-015-0385-y.

126. Ye, J. An extended TOPSIS method for multiple attribute group decision making based on single valued neutrosophic linguistic numbers. J. Intell. Fuzzy Syst. 2015, 28, 247-255.

127. Ye, J. Some aggregation operators of interval neutrosophic linguistic numbers for multiple attribute decision making. J. Intell. Fuzzy Syst. 2014, 27, 2231-2241.

128. Ma, Y.X.; Wang, J.Q.; Wang, J.; Wu, X.H. An interval neutrosophic linguistic multi-criteria group decision-making method and its application in selecting medical treatment options. Neural Comput. Appl. 2017, 28, 2745-2765, doi:10.1007/s00521-016-2203-1.

129. Tian, Z.P.; Wang, J.; Zhang, H.Y.; Chen, X.H.; Wang, J.Q. Simplified neutrosophic linguistic normalized weighted Bonferroni mean operator and its application to multi-criteria decision making problems. Filomat 2015, 30, 3339-3360, doi:10.2298/FIL1508576F.

130. Broumi, S.; Smarandache, F. Single valued neutrosophic trapezoid linguistic aggregation operators based on multi-attribute decision making. Bull. Pure Appl. Sci. Math. Stat. 2014, 33, 135-155.

131. Broumi, S.; Smarandache, F. An extended TOPSIS method for multiple attribute decision making based on interval neutrosophic uncertain linguistic variables. Neutrosoph. Sets Syst. 2015, 8, 22-31.

132. Ye, J. Multiple attribute group decision making based on interval neutrosophic uncertain linguistic variables. Int. J. Mach. Learn. Cybern. 2017, 8, 837-848, doi:10.1007/s13042-015-0382-1.

133. Dey, P.P.; Pramanik, S.; Giri, B.C. An extended grey relational analysis based multiple attribute decision making in interval neutrosophic uncertain linguistic setting. Neutrosoph. Sets Syst. 2016, 11, 21-30.

134. Deli, I.; Broumi, S.; Smarandache, F. On neutrosophic refined sets and their applications in medical diagnosis. J. New Theory 2015, 6, 88-98.

135. Broumi, S.; Deli, I. Correlation measure for neutrosophic refined sets and its application in medical diagnosis. Palest. J. Math. 2016, 5, 135-143.

136. Pramanik, S.; Banerjee, D.; Giri, B.C. TOPSIS approach for multi attribute group decision making in refined neutrosophic environment. In New Trends in Neutrosophic Theory and Applications; Smarandache, F., Pramanik, S., Eds.; Pons Editions: Brussels, Belgium, 2016; pp. 79-91.

137. Pramanik, S.; Banerjee, D.; Giri, B.C. Multi-criteria group decision making model in neutrosophic refined set and its application. Glob. J. Eng. Sci. Res. Manag. 2016, 3, 12-18, doi:10.5281/zenodo.55307. 
138. Mondal, K.; Pramanik, S. Neutrosophic refined similarity measure based on tangent function and its application to multi-attribute decision making. J. New Theory 2015, 8, 41-50.

139. Mondal, K.; Pramanik, S. Neutrosophic refined similarity measure based on cotangent function and its application to multi-attribute decision making. Glob. J. Adv. Res. 2015, 2, 486-494.

140. Mondal, K.; Pramanik, S.; Giri, B.C. Multi-criteria group decision making based on linguistic refined neutrosophic strategy. In New Trends in Neutrosophic Theory and Applications; Smarandache, F., Pramanik, S., Eds.; Pons Editions: Brussels, Belgium; Volume II, in press.

141. Şubaş, Y.; Deli, I. Bipolar neutrosophic refined sets and their applications in medical diagnosis. In Proceedings of the International Conference on Natural Science and Engineering (ICNASE'16), Kilis, Turkey, 19-20 March 2016; pp. 1121-1132.

142. Ye, J. Correlation coefficient between dynamic single valued neutrosophic multisets and its multiple attribute decision-making method. Information 2017, 8, 41, doi:10.3390/info8020041.

143. Majumdar, P.; Samanta, S.K. On similarity and entropy of neutrosophic sets. J. Intell. Fuzzy Syst. 2014, 26, $1245-1252$.

144. Ye, J. Single valued neutrosophic cross-entropy for multi criteria decision making problems. Appl. Math. Model. 2013, 38, 1170-1175.

145. Ye, J. Improved cross entropy measures of single valued neutrosophic sets and interval neutrosophic sets and their multi criteria decision making methods. Cybern. Inf. Technol. 2015, 15, 13-26, doi:10.1515/cait-2015-0051.

(C) 2018 by the authors. Licensee MDPI, Basel, Switzerland. This article is an open access article distributed under the terms and conditions of the Creative Commons Attribution (CC BY) license (http://creativecommons.org/licenses/by/4.0/). 